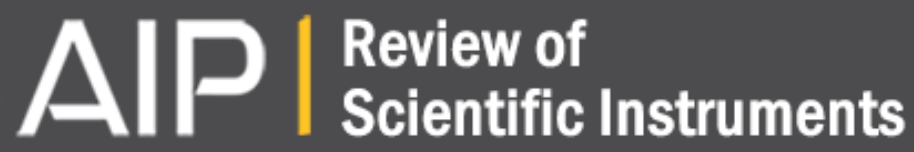

\section{Photoacoustic imaging in biomedicine}

Minghua Xu and Lihong V. Wang

Citation: Review of Scientific Instruments 77, 041101 (2006); doi: 10.1063/1.2195024

View online: http://dx.doi.org/10.1063/1.2195024

View Table of Contents: http://scitation.aip.org/content/aip/journal/rsi/77/4?ver=pdfcov

Published by the AIP Publishing

\section{Articles you may be interested in}

Ultrasound (US) transducer of higher operating frequency detects photoacoustic (PA) signals due to the contrast in elastic property

AIP Advances 6, 025210 (2016); 10.1063/1.4942106

Dedicated 3D photoacoustic breast imaging

Med. Phys. 40, 113301 (2013); 10.1118/1.4824317

High-frequency annular array with coaxial illumination for dual-modality ultrasonic and photoacoustic imaging Rev. Sci. Instrum. 84, 053705 (2013); 10.1063/1.4804636

Optical-resolution photoacoustic microscopy based on two-dimensional scanning galvanometer Appl. Phys. Lett. 100, 023702 (2012); 10.1063/1.3675907

Noninvasive, in vivo imaging of the mouse brain using photoacoustic microscopy J. Appl. Phys. 105, 102027 (2009); 10.1063/1.3116134

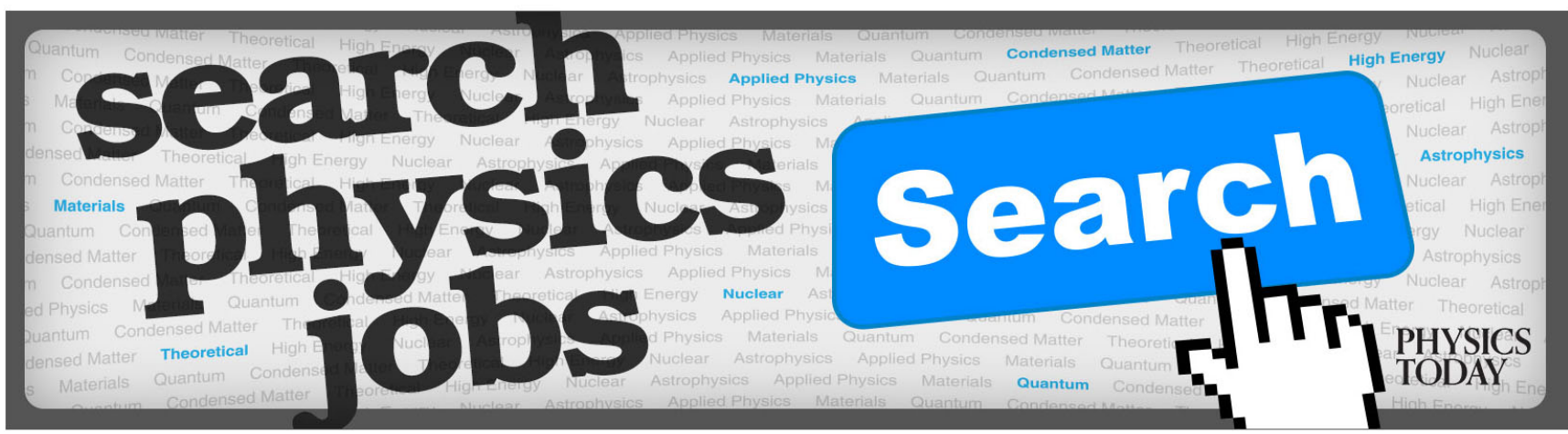




\title{
Photoacoustic imaging in biomedicine
}

\author{
Minghua $\mathrm{Xu}^{\mathrm{a})}$ and Lihong $\mathrm{V}$. Wang ${ }^{\mathrm{b})}$ \\ Optical Imaging Laboratory, Department of Biomedical Engineering, Texas A\&M University, 3120 TAMU, \\ College Station, Texas 77843-3120
}

(Received 15 January 2004; accepted 20 February 2006; published online 17 April 2006)

\begin{abstract}
Photoacoustic imaging (also called optoacoustic or thermoacoustic imaging) has the potential to image animal or human organs, such as the breast and the brain, with simultaneous high contrast and high spatial resolution. This article provides an overview of the rapidly expanding field of photoacoustic imaging for biomedical applications. Imaging techniques, including depth profiling in layered media, scanning tomography with focused ultrasonic transducers, image forming with an acoustic lens, and computed tomography with unfocused transducers, are introduced. Special emphasis is placed on computed tomography, including reconstruction algorithms, spatial resolution, and related recent experiments. Promising biomedical applications are discussed throughout the text, including (1) tomographic imaging of the skin and other superficial organs by laser-induced photoacoustic microscopy, which offers the critical advantages, over current high-resolution optical imaging modalities, of deeper imaging depth and higher absorption contrasts, (2) breast cancer detection by near-infrared light or radio-frequency-wave-induced photoacoustic imaging, which has important potential for early detection, and (3) small animal imaging by laser-induced photoacoustic imaging, which measures unique optical absorption contrasts related to important biochemical information and provides better resolution in deep tissues than optical imaging. () 2006 American Institute of Physics. [DOI: 10.1063/1.2195024]
\end{abstract}

\section{INTRODUCTION}

The photoacoustic (PA) effect is the physical basis for PA imaging; it refers to the generation of acoustic waves by the absorption of electromagnetic (EM) energy, such as optical or radio-frequency (rf) (for simplicity, we will use rf to represent either microwave or $\mathrm{rf}$ waves or both throughout the text) waves. Alexander Graham Bell first reported the observation of sound generated by light in $1880 .{ }^{1}$ Readers are referred to earlier reviews, ${ }^{2-6}$ books and conference proceedings, ${ }^{7-12}$ and original studies for the historical development of PA techniques in various branches of physics, chemistry, biology, engineering, and medicine.

In the last decade, work on photoacoustic imaging in biomedical applications has come a long way. ${ }^{13-17}$ Nonionizing waves, such as short laser or rf pulses, are often used to excite megahertz ultrasound waves, referred to as photoacoustic or thermoacoustic signals, in biological tissues. The motivation for photoacoustic imaging is to combine ultrasonic resolution with high contrast due to light, or rf, absorption. Unlike ionizing $\mathrm{x}$-ray radiation, nonionizing waves pose no health hazard. Unfortunately, however, in the pure optical imaging methodologies, optical scattering in soft tissues degrades spatial resolution significantly with depth. Since ul-

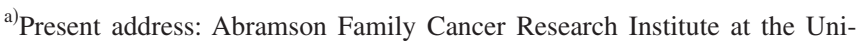
versity of Pennsylvania School of Medicine, Room 627 BRB II/III, 421 Curie Boulevard, Philadelphia, PA 19104-6160.

b) Author to whom all correspondence should be addressed; also at the Department of Biomedical Engineering at Washington University in St. Louis, One Brookings Drive, Campus Box 1097, St. Louis, MO 631304899 as of June 2006; FAX: 979-845-4450; electronic mails: lwang@tamu.edu and lwang@bme.tamu.edu; URL: http://oilab.tamu.edu
}

trasound scattering is two to three orders of magnitude weaker than optical scattering in biological tissues, ${ }^{18}$ ultrasound can provide a better resolution than optical imaging in depths greater than $\sim 1 \mathrm{~mm}$. However, pure ultrasound imaging is based on the detection of the mechanical properties in biological tissues, so its weak contrasts are not capable of revealing early stage tumors. Moreover, ultrasound cannot image either oxygen saturation or the concentration of hemoglobin, to both of which optical absorption is very sensitive. These physiological parameters can provide functional imaging. Likewise, pure rf imaging cannot provide good spatial resolution because of its long wavelength. ${ }^{19}$ Utilizing operating frequencies in the range of $500-900 \mathrm{MHz}$, pure rf imaging can only provide a spatial resolution of $\sim 1 \mathrm{~cm} .{ }^{20}$ The significance of PA imaging is that it overcomes the above problems and yields images of high EM contrast at high ultrasonic resolution in relatively large volumes of biological tissues.

PA imaging can be considered either an ultrasoundmediated EM imaging modality or an ultrasound imaging modality with EM-enhanced contrast. Upon absorption of a short EM pulse, the spatial distribution of the acoustic transient pressure inside the tissue that acts as the initial source for the acoustic waves is simultaneously excited by thermoelastic expansion. The acoustic waves from the initial acoustic source reach the surface of the tissue with various time delays. Ultrasound receivers are placed around the tissues to measure these outgoing acoustic waves, which are further used to determine the initial acoustic source distribution that maps the EM energy deposition functions or absorption properties. The spatial resolution of PA imaging, as well 
as the maximum imaging depth, is scaleable with the detected ultrasonic bandwidth. ${ }^{21}$ For example, PA signals with a $1 \mathrm{MHz}$ bandwidth can provide approximately $1 \mathrm{~mm}$ spatial resolution since the velocity of sound in soft tissues is $\sim 1.5 \mathrm{~mm} / \mu \mathrm{s} .{ }^{18}$ If the bandwidth is increased to $10 \mathrm{MHz}$, approximately $0.1 \mathrm{~mm}$ resolution can be achieved at the expense of ultrasonic penetration.

In a simple case where a wide beam of light pulse heats a layered medium, the detected PA signal replicates the light energy deposition profile throughout the depth. Then, the depth-dependent information of the sample, such as the depth structure and properties (e.g., the absorption coefficient in a nonscattering medium) can be determined directly from the temporal PA signal. For convenience, this imaging configuration is termed PA depth profiling. However, to image more complicated structures, a more complex imaging method referred to as PA tomography (PAT) is preferred. PAT makes use of PA signals measured at various locations around the subject under study. PAT is also called optoacoustic tomography (OAT) or thermoacoustic tomography (TAT), with the term "thermoacoustic" emphasizing the thermal expansion mechanism in the PA generation. OAT refers particularly to light-induced PAT, while TAT is used to refer to rf-induced PAT. Depth profiling can be regarded as onedimensional (1D) PAT. There have been various reviews of optoacoustic imaging in the literature. Oraevsky and Karabutov discussed the generation and detection of optoacoustic profiles and applications of depth profiling for the measurement of tissue optical properties and 1D imaging; ${ }^{22}$ they also presented an analysis of optoacoustic tomography and its applications to the detection of cancer. ${ }^{23}$

From a physical point of view, PAT represents an inverse source problem that belongs to the field of diffraction tomography, due to the diffractive nature of ultrasonic waves. Many imaging concepts and mathematical techniques for other imaging modalities, such as ultrasonic, x-ray, and optical tomographies, can be borrowed for use with PAT. Focused ultrasonic transducers or acoustic lenses can be used directly to form images of the initial pressure distribution. Alternatively, computed tomography (CT), with the measurements often acquired by unfocused ultrasonic transducers, is a more sophisticated method that requires computer-based reconstruction.

This article is intended to provide an overview of PA imaging, including both OAT and TAT. We will start by providing a description of photoacoustics in soft tissues. After that, we will discuss depth profiling, but only briefly, since good coverage is available in previous reviews. ${ }^{22,23} \mathrm{Next}$, we will briefly discuss PA scanning tomography with focused transducers and image forming with acoustic lenses. Then, we will review PA computed tomography in detail. Emphasis throughout will be placed on recent results. Representative works will be summarized with illustrative examples included.

\section{PHOTOACOUSTICS IN TISSUES}

\section{A. EM absorption and penetration}

Electromagnetic energy in the optical (from visible to near-IR) and rf regions is often utilized for PA excitation in soft tissues. This is not only because EM waves in these regions are nonionizing and safe for human use but also because they provide the high contrast and adequate penetration depths ${ }^{18,24-27}$ in biological tissues that are required for various applications. No other EM spectrum seems practical for PA generation in deep tissues. For example, terahertz rays that lie between the above two EM spectra do not penetrate biological tissue well due to water-dominated absorption. In the short-wavelength spectrum below the visible region, such as ultraviolet rays, radiation has high photon energy and, therefore, is harmful to human subjects.

\section{Optical properties}

The optical properties of biological tissues in the visible $(400-700 \mathrm{~nm})$ and near-IR $(700-1100 \mathrm{~nm})$ regions of the EM spectrum are related to the molecular constituents of tissues and their electronic and/or vibrational structures. They are intrinsically sensitive to tissue abnormalities and functions. Optical properties include scattering and absorption. Optical scattering properties can reveal architectural changes in biological tissue at the cellular and subcellular levels, whereas optical absorption properties can be used to quantify angiogenesis and hypermetabolism. Light scattering is quite strong in biological tissues. The reduced or effective scattering coefficient is described by $\mu_{s}^{\prime}=\mu_{s}(1-g)$; where $\mu_{s}$ and $g$ are the scattering coefficient and the anisotropy factor, respectively. In the visible to near-IR region, typically $\mu_{s}$ $\sim 100 \mathrm{~cm}^{-1}$ and $g \sim 0.9$, the absorption coefficients $\mu_{a}$ vary between $\sim 0.1$ and $\sim 10 \mathrm{~cm}^{-1}$ in biological tissues. ${ }^{24}$ Contrast agents, such as indocyanine green (ICG), can be used to increase optical absorption. There is an optical window, lying typically between 700 and $900 \mathrm{~mm}$, that allows light to penetrate relatively deeply up to several centimeters into biological tissues. In general, light propagation in tissues can be described by the radiative transport equation or, with knowledge of the tissue's optical properties, by a Monte Carlo model. Multiple scattering leads to the spreading of light beams and a loss of directionality. Light propagation in deep tissues where multiple scattering prevails approximately follows the diffusion law. Therefore, high-resolution optical imaging modalities, based on ballistic or quasiballistic photons, can image only approximately one photon transport mean free path $(\sim 1 \mathrm{~mm})$ into tissue. Pure optical imaging methods with diffusing light can only achieve a resolution of about $1 \mathrm{~cm}^{25}$ On the other hand, PA imaging actually detects the absorbed photons and can, therefore, image deeper tissues where the diffusion photons are absorbed to generate ultrasound in the $1-50 \mathrm{MHz}$ range. Therefore, higher spatial resolution is possible because ultrasound scattering in tissue is two to three orders of magnitude weaker than optical scattering.

Optical absorption in tissues is a function of the molecular composition. For example, hemoglobin is a constituent in biological tissue that exhibits several absorption bands. ${ }^{25}$ The absorption spectrum of hemoglobin changes when binding occurs. Oxygenated hemoglobin is a strong absorber up to $600 \mathrm{~nm}$ at which point its absorption drops off very steeply, by almost two orders of magnitude, and remains low. The absorption of deoxygenated hemoglobin, however, does not 
drop dramatically; it stays relatively high, although it decreases with increasing wavelengths. The isosbestic point where the two extinction spectra intersect occurs at about $800 \mathrm{~nm}$. The oxygen saturation of hemoglobin is related closely to the metabolic state of lesions and, hence, is an important diagnostic parameter. Rapidly growing (hypermetabolism) cancer cells need additional blood and they gradually develop a dense microvascular network (angiogenesis) around themselves to perpetuate tumor growth. ${ }^{28,29}$ As a consequence, photoacoustic imaging that relies on optical properties can be used to deduce certain physiological parameters, such as the oxygen saturation of hemoglobin and the concentration of hemoglobin, as well as, potentially, to quantify the hallmarks of cancer (including angiogenesis and hypermetabolism), thereby offering earlier cancer detection. More details about the optical properties of biological tissues can be found in Ref. 25 .

\section{2. rf properties}

The rf properties of biological tissues are related to the physiological nature of their electrical properties. The electrical properties ${ }^{26}$ can be described by complex permittivity, $\varepsilon^{*}=\varepsilon^{\prime} \varepsilon_{0}+\sigma /(j \omega)$, or complex conductivity, $\sigma^{*}=\sigma+j \omega \varepsilon^{\prime} \varepsilon_{0}$, where $\sigma$ is the conductivity $(\mathrm{S} / \mathrm{m}) ; \varepsilon^{\prime}$ is the relative permittivity (dimensionless); $\varepsilon_{0}=8.85 \mathrm{pF} / \mathrm{m} \quad$ (permittivity of vacuum); and $\omega$ is the angular frequency. In terms of these properties, the wavelength $\lambda$ of an EM wave in tissue is $\lambda$ $=c_{0} /\left(f \operatorname{Re} \sqrt{\varepsilon^{*} / \varepsilon_{0}}\right)$ and the $1 / e$ penetration depth of the field is $\delta=c_{0} /\left(2 \pi f \operatorname{Im} \sqrt{\varepsilon^{*} / \varepsilon_{0}}\right)$, where Re and Im represent the real and imaginary parts, respectively, and $c_{0}$ is the velocity of the rf wave in vacuo.

In the rf region, such as $0.3-3 \mathrm{GHz}$, EM waves can be readily transmitted through, absorbed, or reflected by biological tissue to varying degrees depending on the body size, tissue properties, and EM frequency; however, little scattering occurs in tissues in this frequency range. ${ }^{27}$ The penetration depth is equal to the reciprocal of the absorption coefficient when scattering and diffraction are ignored. For example, the absorption coefficients of the electric field in fat (low water content) and muscle (high water content) are about 0.1 and $0.9 \mathrm{~cm}^{-1}$, respectively, at $3 \mathrm{GHz}$ and about 0.03 and $0.25 \mathrm{~cm}^{-1}$, respectively, at $300 \mathrm{MHz}$. The most investigated and documented effect of rf power on biological tissues is the transformation of the energy entering the tissues into increased kinetic energy in the absorbing molecules, which produces general heating in the medium. ${ }^{27}$ The two properties that have the strongest effect on the degree of rf absorption are ionic conductivity and the vibration of the dipolar molecules of water and proteins in the biological tissues. ${ }^{27} \mathrm{~A}$ small increase in ionic conductivity or water content in tissue can produce a significant increase in rf absorption.

\section{Safety}

For safety reasons, human exposure to EM radiation must be limited. One of the important technical parameters for safety is the so-called maximum permissible exposure (MPE), which is defined as the level of EM radiation to which a person may be exposed without hazardous effects or biological changes. MPE levels are determined as a function of EM wavelength (or frequency), exposure time, and pulse repetition. The MPE is usually expressed in terms of either radiant exposure in $\mathrm{J} / \mathrm{cm}^{2}$, or irradiance in $\mathrm{W} / \mathrm{cm}^{2}$, for a given wavelength and exposure duration. Exposure to EM energy above the MPE can potentially result in tissue damage. Generally, the longer the wavelength, the higher the MPE; and the longer the exposure time, the lower the MPE.

The IEEE Standard (Std. C95.1, 1999 edition) defines MPE levels, with respect to human exposure to rf fields, from $3 \mathrm{KHz}$ to $300 \mathrm{GHz} .{ }^{30}$ For a rf radiation in the range of $0.3-3 \mathrm{GHz}$ in a controlled environment, MPE $=f / 300 \mathrm{~mW} / \mathrm{cm}^{2}$, where $f$ is the frequency in megahertz. The American National Standard (Z136.1-2000) defines MPE levels for specific laser wavelengths $(180 \mathrm{~nm}-1 \mathrm{~mm})$ and exposure durations. ${ }^{31}$ For example, in the case of skin exposure to a laser beam in the visible and NIR range $(400-1400 \mathrm{~nm}), \quad \mathrm{MPE}=20 C_{A} \mathrm{~mJ} / \mathrm{cm}^{2}$ for a single short pulse with a duration of 1-100 ns, respectively, where $C_{A}$ $=1.0$ in $400-700 \mathrm{~nm}, 10^{2(\lambda-0.7)}$ in $700-1050 \mathrm{~nm}$, and 5.0 in $1050-1400 \mathrm{~nm}$, respectively; $\lambda$ is the wavelength in microns. The above two standards also define formulas to determine the applicable MPE for exposure to repetitive illumination, which is dependent on the wavelength, the pulse repetition frequency, the duration of a single pulse, the duration of any pulse groups, and the duration of the complete exposure.

\section{B. Photoacoustic generation}

Although other generation mechanisms exist, for medical imaging, we are generally interested in using EM pulses to excite transient ultrasonic waves through the thermoelastic mechanism with a low fluence of EM radiation. A sound or stress wave is produced because of the thermoelastic expansion that is induced by a slight temperature rise, typically in the millikelvin range, as a result of the energy deposition inside the biological tissue through the absorption of the incident EM energy. The excited PA signal is locally determined by the EM absorption and scattering properties, the thermal properties, including the thermal diffusivity and thermal expansion coefficient, and the elastic properties of the sample.

The EM absorption property is of primary interest because of the contrast it provides in biological tissues. The thermoelastic mechanism has the following features that make PA techniques amenable for biomedical applications. First, it does not break or change the properties of the biological tissue under study. Second, only nonionizing radiation is used, unlike in x-ray imaging or positron-emission tomography. The nondestructive (noninvasive) and nonionizing nature of PA techniques makes them ideal for in vivo applications. Third, the relationships between PA signals and the physical parameters of biological tissues are well defined. This advantage permits the quantification of various physiological parameters such as the oxygenation of hemoglobin.

To generate PA signals efficiently, two conditions, referred to as thermal and stress confinements, must be met. ${ }^{12}$ The time scale for the heat dissipation of absorbed EM energy by thermal conduction can be approximated by $\tau_{\text {th }}$ 
$\sim L_{p}^{2} / 4 D_{T}$, where $L_{p}$ is the characteristic linear dimension of the tissue volume being heated (i.e., the penetration depth of the EM wave or the size of the absorbing structure). Actually, heat diffusion depends on the geometry of the heated volume, and the estimation of $\tau_{\text {th }}$ may vary. ${ }^{32}$ Upon the absorption of a pulse with a temporal duration of $\tau_{p}$, the thermal diffusion length during the pulse period can be estimated by ${ }^{5,32} \delta_{T}=2 \sqrt{D_{T} \tau_{p}}$, where $D_{T}$ is the thermal diffusivity of the sample, and a typical value for most soft tissues is $D_{T}$ $\sim 1.4 \times 10^{-3} \mathrm{~cm}^{2} / \mathrm{s}$. ${ }^{18}$ The pulse width $\tau_{p}$ should be shorter than $\tau_{\text {th }}$ to generate PA waves efficiently, a condition that is commonly referred to as thermal confinement where heat diffusion is negligible during the excitation pulse. For example, for a rf pulse of $\tau_{p}=0.5 \mu \mathrm{s}, \delta_{T} \approx 0.5 \mu \mathrm{m}$, which is much less than the spatial resolution that most PA imaging systems can achieve. Therefore, the thermal confinement condition is typically met.

Similarly, the time for the stress to transit the heated region can be estimated by $\tau_{s}=L_{p} / c$, where $c$ is the speed of sound. The pulse width $\tau_{p}$ should be shorter than $\tau_{s}$, a condition that is commonly referred to as stress confinement. Under the stress confinement condition, high thermoelastic pressure in the sample can build up rapidly. ${ }^{12}$ For example, to achieve a spatial resolution at $L_{p}=150 \mu \mathrm{m}$, if $c$ $=1.5 \mathrm{~mm} / \mu \mathrm{s}$ and $D_{T} \sim 1.4 \times 10^{-3} \mathrm{~cm}^{2} / \mathrm{s}$, then $\tau_{\text {th }} \sim 40 \mathrm{~ms}$ and $\tau_{s} \sim 100 \mathrm{~ns}$. Hence, $\tau_{p}$ must be less than $100 \mathrm{~ns}$ to guarantee the more stringent stress confinement. When both thermal and stress confinements are satisfied, thermal expansion causes a pressure rise $p_{0}$ that can be estimated by ${ }^{12,23}$

$$
p_{0}=\left(\beta c^{2} / C_{p}\right) \mu_{a} F=\Gamma A,
$$

where $\beta$ is the isobaric volume expansion coefficient in $\mathrm{K}^{-1}$, $C_{p}$ is the specific heat in $\mathrm{J} /(\mathrm{K} \mathrm{kg}), \mu_{a}$ is the absorption coefficient in $\mathrm{cm}^{-1}, F$ is the local light (or rf) fluence in $\mathrm{J} / \mathrm{cm}^{2}, A$ is the local energy deposition density in $\mathrm{J} / \mathrm{cm}^{3}: A=\mu_{a} F$, and $\Gamma$ is referred to as the Grüneisen coefficient expressed as $\Gamma$ $=\beta c^{2} / C_{p}$.

\section{PA propagation and detection}

EM-pulse excited pressure acts as an acoustic source and initiates further acoustic wave propagation in threedimensional (3D) space. For simplicity, the inhomogeneity of acoustic speed in soft tissues is usually neglected in calculating acoustic wave propagation. The speed of sound is relatively constant at $1.5 \mathrm{~mm} / \mu$ s with a small variation of less than $10 \%$ in most soft tissues. ${ }^{18,33}$ If acoustic heterogeneity becomes important, we should resort to a pure acoustic technique, such as ultrasound tomography, to map out the acoustic inhomogeneity for a more accurate calculation of the PA wave propagation.

In the low-megahertz frequency range, ultrasound in soft tissues has the properties of low scattering and deep penetration. ${ }^{18,33}$ The total attenuation results from the combined losses due to both absorption and scattering, while the scatter component accounts for about $10 \%-15 \%$ of the total attenuation. The attenuation of all tissues is temperature and frequency dependent. The frequency dependency of ultrasonic attenuation can be represented by the expression $\mu$ $=a f^{b}$, where $\mu$ is the ultrasonic attenuation coefficient, $a$ and

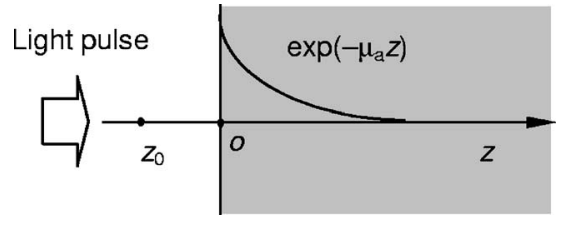

FIG. 1. Diagram of initial pressure distribution.

$b$ are constants, and $f$ is the frequency of ultrasound. ${ }^{18} \mathrm{~A}$ mean value of ultrasound attenuation equals $\sim 0.6 \mathrm{~dB} \mathrm{~cm}^{-1} \mathrm{MHz}^{-1}$ for soft tissues. ${ }^{33}$ The attenuation increases with the frequency and the penetration decreases with the frequency. Typically, $3 \mathrm{MHz}$ might be the maximum frequency for a $15 \mathrm{~cm}$ penetration. ${ }^{33}$ In the high-megahertz frequency range, both scattering and absorption increase tremendously, leading to a markedly decreased penetration depth.

The outgoing ultrasound from the initial source reaches the tissue surface and then can be picked up by an ultrasound transducer. Since it serves only as an acoustic receiver and the emission efficiency is of no importance, the detector for PA measurement can be specially designed for sensitivity. The most often used ultrasound detectors in ultrasound-based imaging are piezoelectric based $;^{33}$ they have low thermal noise and high sensitivity and can provide a wide band of up to $100 \mathrm{MHz}{ }^{23,34}$ The employment of other kinds of sensors, such as those based on optical detection, is also feasible. ${ }^{35-41}$ Optical methods are often based on photoacoustic-pressuredinduced surface displacement ${ }^{35,36,40}$ or refraction index changes, ${ }^{37}$ which means they have the potential for noncontact measurement and rapid monitoring of large areas. ${ }^{38}$ The disadvantages of optical detection relative to piezoelectric detection are lower sensitivity and higher noise level in the range of acoustic frequencies greater than $1 \mathrm{MHz}^{23}$

\section{DEPTH PROFILING}

To begin our discussion of photoacoustic imaging, let us take a simple case of depth profiling or one-dimensional imaging in a layered medium. The temporal shape of a shortpulse excited PA signal in thermal and stress confinements is locally related to the absorption and scattering structure of the tissue sample, and this relationship can sometimes be expressed by an analytic formula. In such a case, the tissue properties (e.g., the absorption coefficient in a nonscattering medium) and structure can be characterized by analyzing the temporal PA signal. ${ }^{42-48}$ Other reviews ${ }^{22,23}$ have discussed 1D or depth-resolved optoacoustic profiling. Here, we only give an example to illustrate the principle of depth profiling.

As shown in Fig. 1, an optically absorbing semi-infinite medium (neglecting optical scattering) in response to a widebeam impulse $\delta(t)$ illumination generates an initial pressure or stress distribution $p_{0}(z)$ as

$$
p_{0}(z)=\Gamma \mu_{a} F_{0} \exp \left(-\mu_{0} z\right),
$$

where $F_{0}$ is the incident laser fluence in $\mathrm{J} / \mathrm{m}^{2}$. The pressure $p_{0}(z)$ serves as the source of an acoustic wave and further prompts two plane waves of equal amplitude to propagate in 
opposite directions along $\pm z$, respectively. Without considering the reflected acoustic wave from the boundary or acoustic attenuation, the PA wave, measured at $z_{0}\left(=-c t_{0}\right)$, is

$$
\begin{aligned}
p\left(z_{0}, t\right)= & \frac{1}{2} \Gamma \mu_{a} F_{0} \exp \left[-\mu_{a}\left(c t+z_{0}\right)\right]=\frac{1}{2} \Gamma \mu_{a} F_{0} \exp [ \\
& \left.-\mu_{a} c\left(t-t_{0}\right)\right], \quad t>t_{0} .
\end{aligned}
$$

The absorption coefficient $\mu_{a}$ can be determined from the amplitude $\Gamma \mu_{a} F_{0} / 2$, if the detection system is calibrated for absolute measurement or from the exponential slope of the PA wave through fitting the curve with $\exp \left(-\mu_{a} c t\right)$. Since it is easier to measure the relative profile than the absolute amplitude, fitting the slope is more reliable. For a strong scattering medium, a more complex expression is available, where the exponential decay term in the diffusion regime is determined by the effective attenuation coefficient $\mu_{\text {eff }}$ instead of $\mu_{a}$.

Based on the above concept, depth profiling is able to characterize tissue optical properties. For example, Oraevsky et $a l^{43}$ utilized the time-resolved detection of laser-induced acoustic transients to determine the optical properties (absorption, scattering, and attenuation coefficients) of various media, including bovine liver, canine prostate, and human fibrous atheroma; Köstli et al. ${ }^{46}$ used the PA method to measure the effective attenuation coefficients of cartilage and chicken breast in the infrared light range.

For a multilayered sample, each layer creates a portion of the temporal profile of the PA signal from which the value of the absorption or attenuation coefficient of each layer can be determined by piecewise exponential fitting. ${ }^{44,45}$ In the fitting process, the temporal profile of the laser pulse can be taken into account although the pulse width is typically quite small. In general, if the absorption or attenuation coefficient is a continuous function of depth in the sample, a reconstruction algorithm is required to extract the absorption information from the detected PA temporal signals. Viator et al. ${ }^{47}$ demonstrated this approach based on Beer's law.

However, except for phantom samples ${ }^{44,45,47}$ the relative measurement error in these experiments ${ }^{43,46}$ is around $10 \%$ because of the property variations in tissue samples. Besides, the deduction of optical properties from PA signals in the range of the acoustic frequencies, where diffraction and attenuation are not negligible, can be complicated. ${ }^{43}$ If the light penetration depth is small compared with the effective penetration depth of the acoustic wave, the acoustic attenuation decreases only the amplitude of the measured PA signal and does not alter its replication of the profile of the light distribution. Nevertheless, when the optical attenuation coefficient is similar to that of the acoustic attenuation, both the amplitude and exponential slope of the initial pressure or stress can change during acoustic-wave propagation in the medium. The diffraction of acoustic waves is prominent when the laser beam diameter is comparable to the light penetration depth.

\section{SCANNING TOMOGRAPHY}

\section{A. Principle}

Photoacoustic scanning tomography is often similar to $B$-mode ultrasonography. Figure 2(a) shows a diagram of

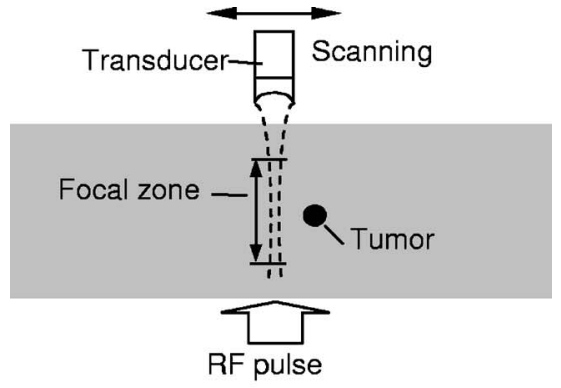

(a)

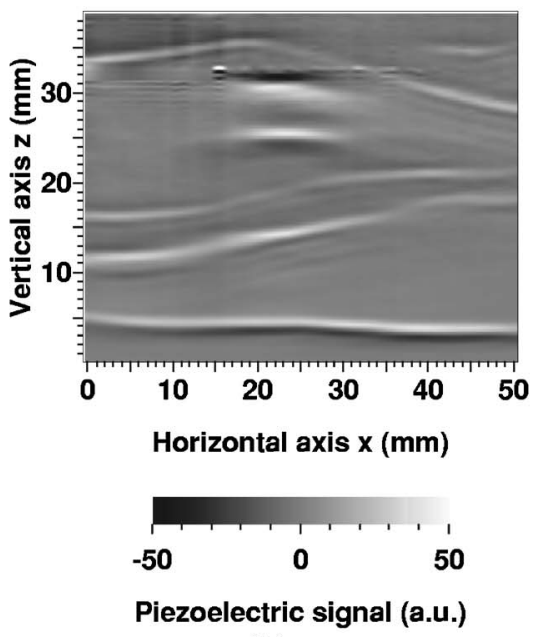

(b)

FIG. 2. (a) Diagram of thermoacoustic scanning tomography. (b) Thermoacoustic image of a phantom sample: a piece of muscle buried in several layers of fat.

scanning tomography in the forward detection mode. A focused ultrasound transducer scans along the tissue surface, and analogous to an ultrasonic $A$-line or $A$-scan, each detected time-resolved signal upon a pulsed-EM excitation can be converted into a 1D image along the acoustic axis of the transducer. Combining multiple $A$-scan images acquired sequentially from various positions on the same plane forms cross-sectional images. The axial resolution along the acoustic axis is dependent on both the width of the radiation pulse and the width of the impulse response of the transducer. The lateral resolution is determined by the focal diameter of the ultrasonic transducer and the center frequency of the received PA signals. In this imaging configuration, the imaging zone is limited by the focal zone of the transducer. ${ }^{49}$ Outside the focal zone along the acoustic axis, the detection sensitivity and image resolution decrease greatly. An alternative configuration of PA scanning tomography is analogous to the $C$-scan mode in ultrasonography, in which a cross-sectional image at a certain image depth is formed, and then slices imaged at different depths can be stacked together to form a 3D image.

To achieve a better signal-to-noise ratio (SNR), a highenergy pulse is preferred, since the amplitude of a PA signal is proportional to the absorbed EM energy. However, for safety reasons, the pulse energy is limited. A focused transducer can detect a PA signal with a high SNR because of its large numerical aperture. Therefore, a single EM pulse is 
usually able to provide a scan line without the necessity of averaging data from multiple shots. However, the EM-pulse repetition frequency limits the scanning speed.

\section{B. rf-based scanning tomography}

A few researchers ${ }^{50-52}$ have demonstrated rf-induced PA imaging by scanning a focused ultrasonic transducer. The pulse duration of the rf source used is often in the range of $0.1-1 \mu$ s, which can excite ultrasound up to several megahertz since the bandwidth of the PA signal approximates the reciprocal of the EM pulse width. The megahertz signal can provide axial spatial resolution in millimeters or submillimeters through a multicentimeter thick tissue. Hence, it is suitable for imaging large samples such as the human breast. Figure 2(b) shows a microwave-induced thermoacoustic image of a phantom sample, ${ }^{51}$ in which the boundaries of the tissues are clearly imaged. It indicates that rf can easily penetrate multiple centimeters through biological tissue to reach deep tumors.

\section{Laser-based microscopic imaging}

PA imaging with pulsed-light excitation can operate in a way that is similar to rf-based scanning tomography. ${ }^{53}$ However, PA imaging with a laser can be scaled down for microscopic imaging. A laser system can easily generate laser pulses with a pulse energy of $100 \mathrm{~mJ}$ and a pulse duration of 10 ns or shorter, which can sufficiently excite PA signals at high frequencies up to $100 \mathrm{MHz}$ in large-area soft tissues with a good SNR. Therefore, laser-based PA scanning tomography can perform microscopic imaging with an axial resolution of $30 \mu \mathrm{m}$ or less, which means it has potential for applications in direct imaging of the skin and other superficial organs or imaging endoscopically in the gastrointestinal (GI) tract.

Oraevsky and Karabutov ${ }^{23}$ demonstrated that optoacoustic microscopy was capable of imaging and distinguishing early stages of squamous-cell carcinoma in the oral mucous of golden hamsters in vivo. The imaging system they used, termed confocal optoacoustic microscopy imaging, is shown in Fig. 3(a), where both the ultrasound detection and the excitation light source are focused on the same spot. ${ }^{23,54}$ The pulsed light is delivered via optical fiber and focused by a condenser [gradient index (GRIN)] lens onto the tissue surface through an optoacoustic (OA) lens. The induced ultrasound propagates backward through the OA lens onto a ringshaped piezoelectric film. This bright-field design suffers from the strong photoacoustic waves that are emitted from optical absorbers near the surface, the acoustic reverberations from which can potentially overshadow the much weaker photoacoustic signals from structures deep in the tissue.

To prevent the occurrence of such overshadow problems, a reflection-mode microscopic photoacoustic imaging technique that uses dark-field illumination, as in dark-field microscopy, was recently reported by Maslov et al. ${ }^{55}$ as shown in Fig. 3(b). In this design, the light comes onto the tissue surface in a doughnut or small ring shape, and then the diffusion photons transporting to the imaging axis are absorbed to generate ultrasound. As a consequence, this design par-

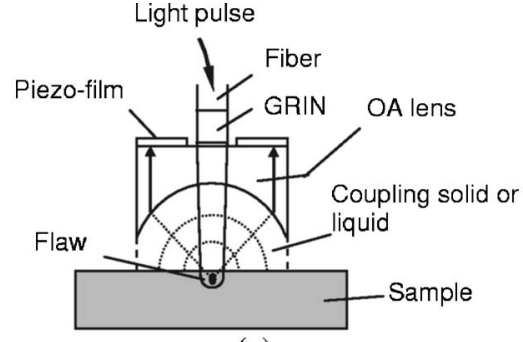

(a)

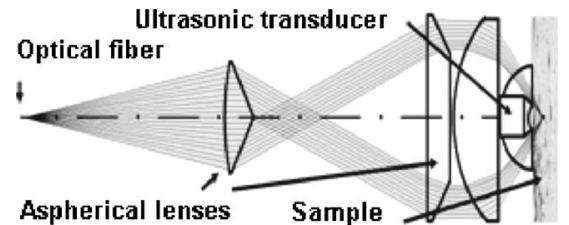

(b)

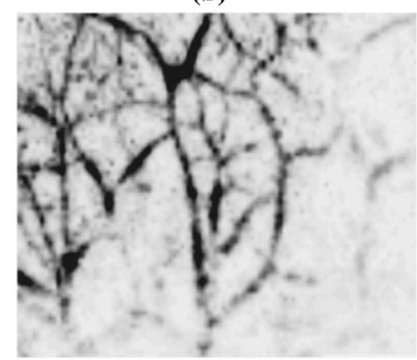

(c)

FIG. 3. (a) Diagram of a bright-field confocal photoacoustic microscope in the backward detection mode. (b) Schematic of the photoacoustic sensor of a dark-field reflection-mode photoacoustic microscope. (c) Photoacoustic image of vascular distribution in rat skin.

tially averages out the shadows of superficial heterogeneity in the image and also reduces the potentially strong interference of the extraneous photoacoustic signals from the superficial paraxial areas. In their recent system, the lateral resolution was as high as $45 \mu \mathrm{m}$ in the tissue phantoms. The maximum imaging depth was at least $3 \mathrm{~mm}$. Further improvement of the image resolution by increasing the ultrasonic frequency is possible at the cost of imaging depth. An in situ photoacoustic image similar to a $C$-scan image (100 $\times 100$, pixels; $0.1 \mathrm{~mm}$ step size) is shown in Fig. 3(c), in which the vascular distribution in rat skin was clearly imaged.

Because of the strong light scattering, PA imaging resolution beyond one optical transport mean free path in tissue is determined primarily by the ultrasonic detection parameters. To provide high resolution, the acoustic detector must have a wide bandwidth and a large numerical aperture (NA). However, increasing the ultrasonic frequency too much can result in an undesirably small penetration depth because the ultrasonic attenuation in tissues, $0.7-3 \mathrm{~dB} \mathrm{~cm}^{-1} \mathrm{MHz}^{-1}$ for human skin, ${ }^{56}$ for example, increases linearly with the frequency. Therefore, a large NA is essential for the desired resolution.

In summary, PA microscopy has critical advantages over other optical-contrast imaging methods, including current high-resolution optical imaging techniques such as confocal microscopy and optical coherence tomography (OCT). These optical imaging techniques can image only approximately one transport mean free path $(\sim 1 \mathrm{~mm})$ into tissue because 


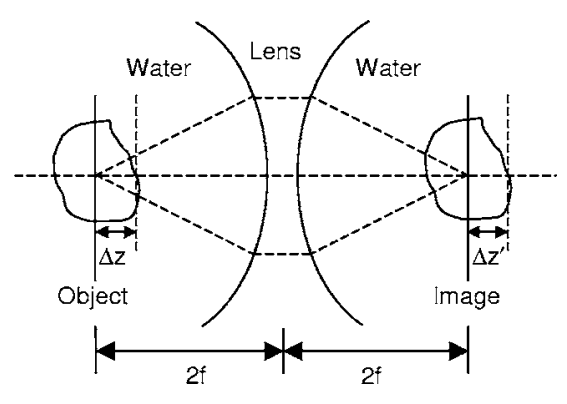

FIG. 4. Acoustic lens system with a focal length of $f$.

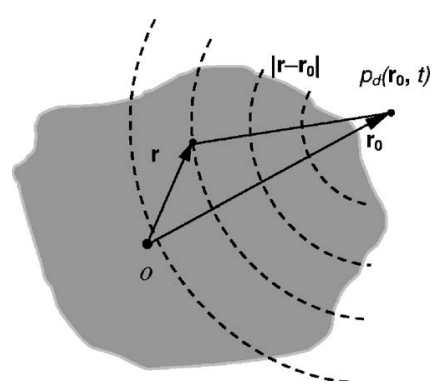

FIG. 5. Diagram of photoacoustic measurement at $\mathbf{r}_{0}$. they depend on ballistic or quasiballistic photons. In addition, they are sensitive to the backscattering that is related to tissue morphology, but they are insensitive to the optical absorption that is related to important biochemical information. PA microscopy imaging does not rely on ballistic or quasiballistic photons and can, therefore, penetrate deeper. Further, it provides high optical-absorption contrast while it maintains high ultrasonic resolution due to the low scattering of megahertz ultrasound. Consequently, structures with high optical absorption coefficients, such as blood vessels, can be imaged clearly by PA microscopy.

The emergence of PA microscopy in the early 2000s offers a novel opportunity for detecting and imaging skin cancer (e.g., melanoma) in vivo. The precise imaging of lesion size, location, and surrounding abnormal vascularity will definitely benefit tumor staging, surgery, and treatment.

\section{IMAGE FORMING WITH ACOUSTIC LENSES}

An acoustic lens can be used to diverge or converge acoustic waves in a manner analogous to an optical lens refracting light. Also similar to an optical imaging system, an acoustic lens is able to image the initial PA pressure distribution in an optically turbid medium onto an image space in an optically transparent medium in which the initial pressure distribution can be directly measured in real time without the necessity of scanning detections or computational reconstructions. For example, a two-dimensional (2D) ultrasonic detector array with multiple small elements can be inserted into an image space to get a slice of the focused image. Because of the slow speed of ultrasound relative to light, images should be taken after the EM-pulse illumination when the ultrasound is focused on the image space, forming an approximate replica of the original PA pressure distribution in the clear medium.

Recently, Niederhauser et al. ${ }^{57}$ proposed an optical darkfield stereo imaging system using a $30 \mathrm{~ns}$ flash illumination light source to capture a snapshot of pressure-induced refraction index changes in a water container at a predetermined time after the original laser pulse. In their system, the acoustic lens system consisted of a "biconcave" aspheric aluminum lens covered on both sides with distilled water, as shown in Fig. 4. The aluminum surfaces were coated with a $40 \mu \mathrm{m}$ antireflex parylene coating to minimize acoustic reflection at the boundary. The $4 f$ lens configuration was chosen (46 mm water, $22 \mathrm{~mm}$ aluminum, and $46 \mathrm{~mm}$ water) to provide a unit magnification both laterally and axially when the object is located at $2 f$ and has a size much smaller than
$2 f$. Based on the lens-imaging formula $1 /(2 f-\Delta z)+1 /(2 f$ $\left.+\Delta z^{\prime}\right)=1 / f$, we have $\Delta z^{\prime}=\Delta z /(1-\Delta z / f)$ and $\Delta z^{\prime} \approx \Delta z$ when $\Delta z \ll f$. Therefore, any small displacement of the object plane by a distance $\Delta z$ away from $2 f$ results in a displacement of the corresponding focused image plane by the same value. In this case, the acoustic propagation time from each object plane to the corresponding focused image plane remains the same, independent of the exact individual object position. This results in a perfect $3 \mathrm{D}$ pressure image at a fixed time that equals the acoustic propagation time from the object plane to the corresponding imaging plane. The pressure image in this case is, therefore, identical to the initial pressure distribution except for small alterations introduced by finite aperture and lens aberrations.

\section{COMPUTED TOMOGRAPHY}

\section{A. Introduction}

A majority of recent works have focused on reconstruction-based PAT, which provides more flexibility in dealing with measured PA signals than do the image forming methods with focused transducers or focused lenses that have fixed imaging regions. Technically, each temporal PA signal, measured at various detection positions, provides onedimensional radial information about the PA source relative to the detector position; 2D surface scans offer other 2D lateral information about the PA source. Combining the temporal and spatial measurements affords sufficient information for a complete reconstruction of a 3D PA source. Because the PA signal received by each ultrasound detector is the integral of the ultrasound waves over the sensing aperture of the detector, the reconstruction algorithms depend on the detector apertures as well as the scanning geometries. Small-aperture detectors are often used to approximate point detectors, which receive PA signals originating from spherical shells, centered at each point detector, with radii determined by the acoustic times of flight (Fig. 5). Large-aperture detectors need different reconstruction algorithms. Recently, a reconstruction method based on measurements with large planar detectors was presented: ${ }^{58}$ this method is closely related to the standard Radon transform of the energy deposition function.

In Sec. VI B, we will introduce the inverse source problem. Then, we will review reconstruction based on pointdetector measurements. Because of the 3D nature of acoustic waves, we will review 3D algorithms and methods with point detectors in Sec. VI C, in which we will cite some of 
the related literature about 2D reconstructions. In Sec. VI D, we will discuss spatial resolution. Finally, we will introduce a reconstruction method using large planar receivers in Sec. VI E.

\section{B. Inverse source problem}

In response to a heat source, $H(\mathbf{r}, t)$, the pressure, $p(\mathbf{r}, t)$, at position $\mathbf{r}$ and time $t$ in an acoustically homogeneous liquidlike medium obeys the following wave equation (ignoring thermal diffusion and kinematic viscosity): ${ }^{5,6,12,59}$

$$
\nabla^{2} p(\mathbf{r}, t)-\frac{1}{c^{2}} \frac{\partial^{2}}{\partial t^{2}} p(\mathbf{r}, t)=-\frac{\beta}{C_{p}} \frac{\partial}{\partial t} H(\mathbf{r}, t),
$$

where $H(\mathbf{r}, t)$ is a heating function defined as the thermal energy deposited by the EM radiation per time per volume $\left[C_{p}\right.$ and $\beta$ are defined after Eq. (1)]. The validity of the above equation requires $L_{p} \gg \sqrt{D_{T} \tau_{p}}$ (i.e., thermal confinement: $\tau_{p} \ll \tau_{\text {th }}$ ); $L_{p} \gg D_{T} / c$ (i.e., $\tau_{s} \ll \tau_{\text {th }}$ ) and $c^{2} \tau_{p} \gg D_{T}$ (i.e., stress propagation length $\gg$ heat diffusion length: $c \tau_{p}$ $\gg \sqrt{D_{T} \tau_{p}}$ ); and $c^{2} \tau_{p} \gg \eta / \rho_{0}$ where $\eta / \rho_{0}$ is the kinematic viscosity of the liquid. ${ }^{6}$ Under the above conditions, the initially excited acoustic stress or pressure is determined by the local EM absorption.

The forward solution, based on the free-space Green's function, can be found in the physics or mathematics literature. ${ }^{60-62}$ In general, the solution to Eq. (4) in the time domain can be expressed by

$$
p(\mathbf{r}, t)=\left.\frac{\beta}{4 \pi C_{p}} \iiint \frac{d^{3} r^{\prime}}{\left|\mathbf{r}-\mathbf{r}^{\prime}\right|} \frac{\partial H\left(\mathbf{r}^{\prime}, t^{\prime}\right)}{\partial t^{\prime}}\right|_{t^{\prime}=t-\left|\mathbf{r}-\mathbf{r}^{\prime}\right| / c} .
$$

The heating function can be written as the product of a spatial absorption function and a temporal illumination function under the condition of thermal confinement,

$$
H(\mathbf{r}, t)=A(\mathbf{r}) I_{e}(t) .
$$

Then, Eq. (5) can be rewritten as a convolution between the temporal profile $I_{e}(t)$ and the acoustic wave form $p_{\delta}(\mathbf{r}, t)$ that is excited by an infinitely short pulse $\delta(t)$,

$$
p_{e}(\mathbf{r}, t)=\int_{-\infty}^{+\infty} I_{e}(t-\tau) p_{\delta}(\mathbf{r}, \tau) d \tau
$$

where

$$
p_{\delta}(\mathbf{r}, t)=\frac{\partial}{\partial t}\left[\frac{1}{4 \pi} \iint_{\left|\mathbf{r}-\mathbf{r}^{\prime}\right|=c t} p_{0}\left(\mathbf{r}^{\prime}\right) d \Omega^{\prime}\right],
$$

where $d \Omega^{\prime}$ is the solid-angle element of vector $\mathbf{r}^{\prime}$ with respect to the point at $\mathbf{r}$; and $p_{0}(\mathbf{r})$ is the initial pressure excited by a $\delta(t)$ EM source, computed by $p_{0}(\mathbf{r})=\Gamma(\mathbf{r}) A(\mathbf{r})$, which acts as the source of the propagating acoustic wave.

For simplicity, a $\delta(t)$ EM source is assumed and the PA signal is detected at position $\mathbf{r}_{0}$ by a point detector (Fig. 5),

$$
p_{d}\left(\mathbf{r}_{0}, t\right)=\frac{\partial}{\partial t}\left[\frac{t}{4 \pi} \iint_{\left|\mathbf{r}_{0}-\mathbf{r}\right|=c t} p_{0}(\mathbf{r}) d \Omega\right],
$$

where $d \Omega$ is the solid-angle element of vector $\mathbf{r}$ with respect to the point at $\mathbf{r}_{0}$. Then, the key to the inverse algorithm in
PAT is to reconstruct the initial source $p_{0}(\mathbf{r})$ from the measured data $p_{d}\left(\mathbf{r}_{0}, t\right)$.

\section{Algorithms and methods}

\section{Overview}

a. Radon transform. The projections on the detectors are represented by the integrals over the spherical shells as shown in Eq. (9), in contrast to line integrals in straight-ray tomography such as x-ray CT. The development of an exact algorithm based on the solution of Eq. (9) has serious mathematical difficulties. Therefore, some researchers have applied approximations of the well-known standard Radon transform to PAT reconstruction. For convenience, Eq. (9) is rewritten as

$$
F\left(\mathbf{r}_{0}, t\right)=\frac{4 \pi}{t} \int_{0}^{t} p_{d}\left(\mathbf{r}_{0}, t\right) d t=\iint_{\left|\mathbf{r}_{0}-\mathbf{r}\right|=c t} p_{0}(\mathbf{r}) d \Omega .
$$

If the object is enclosed in the center region by a spherical measurement surface and its size is much smaller than the enclosed volume, the spherical shells over which the surface integral in Eq. (10) is computed approximate the planes, and Eq. (10) approximates the Radon transform used in X-ray CT.

Based on the above concept, Kruger and co-workers ${ }^{63-65}$ suggested a filtered back-projection algorithm under the spherical measurement geometry, i.e., the inverse of Eq. (10) approximating

$$
p_{0}(\mathbf{r}) \approx-\frac{1}{2 \pi} \int_{S_{0}} \int \frac{d S_{0}}{r_{0}^{2}}\left[t \frac{\partial p_{d}\left(\mathbf{r}_{0}, t\right)}{\partial t}+2 p_{d}\left(\mathbf{r}_{0}, t\right)\right]_{t=\left|\mathbf{r}-\mathbf{r}_{0}\right| / c},
$$

where $d S_{0}$ is the detector element at $\mathbf{r}_{0}$. Liu ${ }^{66}$ derived an expression identical to Eq. (11) based on what he called a $p$ transform. Other researchers such as Andreev et al. did numerical simulations based on 2D (Ref. 67) or 3D (Ref. 68) Radon transform approximations, and Xu et al. ${ }^{69}$ tested a 2D Radon transform approximation with a Hilbert transform.

In general, the Radon transform approximation provides a satisfactory reconstruction for an object located near the center of the spherical (or circular) detection geometry. However, the fact that this approximation does not hold when the source deviates from the center of the spherical geometry limits its application. Significant reconstruction artifacts occur when the integration spherical shells are far different from the planar surfaces.

Finally, it must be pointed out that PAT mathematically belongs to the generalized spherical Radon transform. Mathematicians have obtained an inverse formula for the spherical geometry, which will be discussed in Sec. VI C 3.

b. Back projection. Algorithms for ultrasound imaging, such as back projection (delay and sum) and synthetic aperture, have also been borrowed for PAT reconstruction. For example, Hoelen and de Mul and co-workers ${ }^{70,71}$ constructed a time-domain delay-and-sum focused beam-forming algorithm to locate the PA sources in a sample in a planar scan configuration; Köstli et al. ${ }^{72}$ reported an image reconstruction of detected 2D pressure distributions using back projection. Feng et al. ${ }^{73}$ applied a synthetic-aperture method to 
linear-scanning microwave-induced thermoacoustic tomography in biological tissues. Liao et $a .^{74}$ reported on a study of optoacoustic imaging with synthetic aperture focusing and coherence weighting. Yin et al. ${ }^{75}$ used a phase-controlled focus algorithm in their fast photoacoustic imaging system with a multielement linear transducer array.

For the spherical and cylindrical geometries, $\mathrm{Xu}$ et $a l .^{76,77}$ approximated the rigorous Fourier-domain reconstruction formulas (detailed later) to the so-called modified back-projection formula as

$$
\begin{aligned}
p_{0}(\mathbf{r}) \approx & -\frac{1}{2 \pi} \int_{S_{0}} \int \frac{d S_{0}}{\left|\mathbf{r}-\mathbf{r}_{0}\right|^{2}}\left[\mathbf{n}_{0}^{s}\left(-\mathbf{n}_{0}\right)\right] \\
& \times\left[t \frac{\partial p_{d}\left(\mathbf{r}_{0}, t\right)}{\partial t}\right]_{t=\left|\mathbf{r}-\mathbf{r}_{0}\right| / c},
\end{aligned}
$$

where $\mathbf{n}_{0}^{s}$ is the normal of surface $S_{0}$ pointing to the source and $\mathbf{n}_{0}=\mathbf{r}_{0} / r_{0}$. The formula for the planar geometry replaces $2 \pi$ by $\pi$ in Eq. (12). In the approximation, it is assumed that the distances between the PA sources and the detectors are much greater than the wavelengths of the PA signals that are useful for imaging. The modified back-projection formula indicates that in 3D reconstruction, the back-projection quantity is related to the first derivative of the acoustic pressure, rather than simply to the acoustic pressure itself. A weighting factor $t$ compensates for the $1 / t$ attenuation of a spherical pressure wave as it propagates through a homogeneous medium. The contribution to a reconstruction point $P$ from an element of receiving area $d S_{0}$ is proportional to the subtended solid angle of this element $d S_{0}$ when viewed from the point $P$. The solid angle is inversely proportional to the square of the distance between the receiving element $d S_{0}$ and the point $P$. Hence, the modified back-projection formula of Eq. (12) is more general than the Radon transform approximation formula of Eq. (11).

Recently, exact back-projection formulas for the spherical, planar and cylindrical geometries have been reported, which will be detailed in Sec. VI C 3.

c. Fourier- and time-domain algorithms. Recently, analytical algorithms have been derived both in Fourier-domain and time-domain for reconstruction of both TAT and PAT. These algorithms are exact for the full-view data and can serve as a basis for reconstruction of TAT and PAT. We defer discussion of these methods in Secs. VI C 2 and VI C 3, respectively, where we discuss them in detail.

d. Other methods. In principle, Eqs. (9) or (10) can be rewritten in its discrete form as

$$
M \cdot P_{0}=D,
$$

where matrix $P_{0}$ represents the unknown initial pressure, matrix $D$ represents the measured PA signals, and the sensitivity matrix $M$ consists of the known coefficients linking $P_{0}$ to $D$. Then, the standard techniques for solving a linear equation system can be used to compute $P_{0}$. For example, Paltauf et al. ${ }^{78}$ studied an iterative reconstruction algorithm to minimize the error between the measured signals and the theoretical signals calculated from the reconstructed image; $\mathrm{Xu}$ and Wang $^{79}$ also studied an iterative algorithm based on the truncated conjugate gradient (TCG) method. Compared with the approximation methods discussed in Secs. VI C $1 \mathrm{a}$ and VI C $1 \mathrm{~b}$, the iterative method may give a more accurate result. However, the iterative method's multiple steps take more computation time since each step takes an amount of time comparable to all of the steps in the other methods. In addition, large objects require more computer memory for storage of the discrete matrix.

In addition, Zhulina ${ }^{80}$ developed another interesting algorithm based on an optimal statistical approach. The essence of this algorithm includes (1) the summing of all signals in the image plane with the transform from the time coordinates of the signals to the spatial coordinates of the image and (2) the optimal spatial filtration of this sum. Anastasio et al. ${ }^{81}$ reported on half-time reconstruction approaches; they revealed that half-time reconstructions permit the explicit control of statistically complementary information that can result in the optimal reduction of image variances. They also demonstrated that half-time reconstructions can mitigate image artifacts due to the heterogeneous acoustic properties of an object. Zhang et al. ${ }^{82}$ presented weighted expectation maximization reconstruction algorithms, in which they demonstrated that suitable choices of weighted algorithms can effectively mitigate image artifacts that are attributable to the temporal truncation of the measured data.

\section{Fourier-domain algorithms}

a. Spherical geometry. Based on the assumption of a constant sound speed, $\mathrm{Xu}$ and Wang referred to mathematical techniques for ultrasonic reflectivity imaging ${ }^{83}$ and reported an exact Fourier-domain reconstruction for the spherical geometry. $^{76}$

Taking the following Fourier transform with respect to variable $\bar{t}=c t$,

$$
\tilde{p}_{d}\left(\mathbf{r}_{0}, k\right)=\int_{-\infty}^{+\infty} p_{d}\left(\mathbf{r}_{0}, \bar{t}\right) \exp (i k \bar{t}) d \bar{t},
$$

where $k=\omega / c=2 \pi f / c$ with frequency $f$. Equation (9) can be rewritten in the frequency domain as

$$
\tilde{p}_{d}\left(\mathbf{r}_{0}, k\right)=-i k \iiint d^{3} r p_{0}(\mathbf{r}) \tilde{G}_{k}\left(\mathbf{r}_{0}, \mathbf{r}\right),
$$

where the Green's function $\widetilde{G}_{k}\left(\mathbf{r}_{0}, \mathbf{r}\right)=\exp \left(i k\left|\mathbf{r}_{0}-\mathbf{r}\right|\right) /\left(4 \pi \mid \mathbf{r}_{0}\right.$ $-\mathbf{r})$ ) represents a monochromatic spherical acoustic wave emanating from a point source.

We denote the spherical measurement surface $\mathbf{r}_{0}$ $=\left(r_{0}, \theta_{0}, \varphi_{0}\right)$ in the spherical polar coordinates $\mathbf{r}=(r, \theta, \varphi)$ [Fig. 6(a)], where $\theta$ is the polar angle from the $z$ axis and $\varphi$ is the azimuth angle in the $x y$ plane from the $x$ axis. The sample under study lies inside the sphere, i.e., $p_{0}(\mathbf{r})$ $=p_{0}(r, \theta, \varphi)$ where $r<r_{0}$ and $p_{0}(\mathbf{r})=0$ when $r>r_{0}$. The exact reconstruction formula for $p_{0}(\mathbf{r})$ can be written as ${ }^{76}$

$$
\begin{aligned}
p_{0}(\mathbf{r})= & \frac{1}{2 \pi^{2}} \iint_{\Omega_{0}} d \Omega_{0} \int_{0}^{\infty} d k \tilde{p}_{d}\left(\mathbf{r}_{0}, k\right) \\
& \times \sum_{i=0}^{\infty} \frac{(2 l+1) j_{l}(k r)}{h_{l}^{(1)}\left(k r_{0}\right)} P_{l}\left(\mathbf{n}_{0} \cdot \mathbf{n}\right),
\end{aligned}
$$

where $d \Omega_{0}=\sin \theta_{0} d \theta_{0} d \varphi_{0} ; \mathbf{n}=\mathbf{r} / r$ and $\mathbf{n}_{0}=\mathbf{r}_{0} / r_{0}$ are unit vec- 


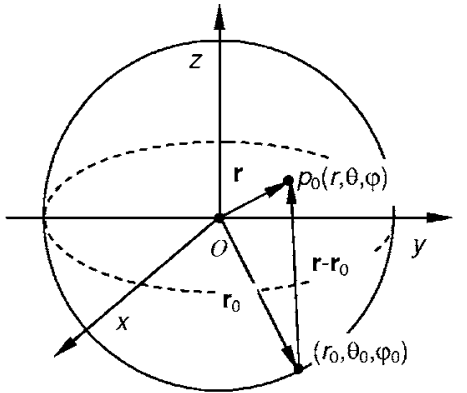

(a)

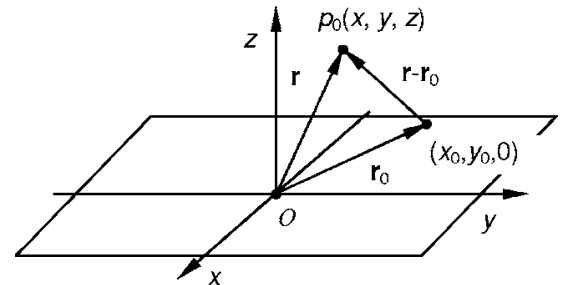

(b)

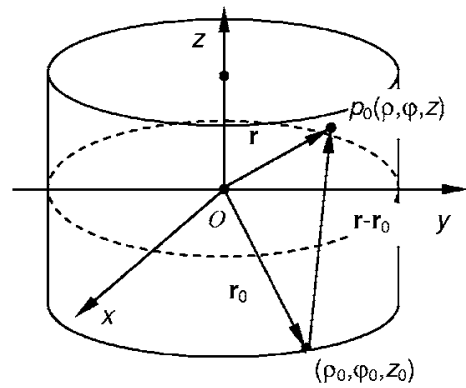

(c)

FIG. 6. Diagram of measurement configurations: (a) spherical geometry, (b) planar geometry, and (c) cylindrical geometry.

tors; $j_{l}(\cdot), h_{l}^{(1)}(\cdot)$, and $P_{l}(\cdot)$ are a spherical Bessel function of the first kind, a spherical Hankel function of the first kind, and a Legendre polynomial function, respectively. If the source $p_{0}(\mathbf{r})$ and the measurement $\tilde{p}_{d}\left(\mathbf{r}_{0}, k\right)$ are expanded in spherical harmonics as

$$
\begin{aligned}
p_{0}(r, \theta, \varphi)= & \frac{1}{2 \pi^{2}} \sum_{l=0}^{+\infty} \sum_{m=-l}^{+l}(-i)^{l} Y_{l}^{m^{*}}(\theta, \varphi) \\
& \times \int_{0}^{+\infty} k^{2} d k j_{l}(k r) \widetilde{p}_{0 l}^{m}(k),
\end{aligned}
$$

and

$$
\tilde{p}_{d}\left(\theta_{0}, \varphi_{0}, k\right)=\sum_{l=0}^{+\infty} \sum_{m=-l}^{+l} \widetilde{q}_{l}^{m}(k) Y_{l}^{m^{*}}\left(\theta_{0}, \varphi_{0}\right),
$$

where the symbol ${ }^{*}$ denotes the complex conjugate, then the relationship between the source distribution and the measured data can be expressed by

$$
\tilde{p}_{0 l}^{m}(k)=\frac{(+i)^{l} 4 \pi \widetilde{q}_{l}^{m}(k)}{k^{2} h_{l}^{(1)}\left(k r_{0}\right)} .
$$

The imaging reconstruction follows in three steps: (1) take the spherical harmonics expansion of $\tilde{p}_{d}\left(\theta_{0}, \varphi_{0}, k\right)[$ inverse of Eq. (18)] to find the decomposition $\widetilde{q}_{l}^{m}(k)$ of $m$ and $l$ as a function of $k,(2)$ compute $\widetilde{p}_{0 l}^{m}(k)$ from $\widetilde{q}_{l}^{m}(k)$ based on
Eq. (19), and (3) take the Hankel transform over $k$ of $\widetilde{p}_{0 l}^{m}(k)$ and then the summation, i.e., Eq. (17), to find the initial pressure $p_{0}(r, \theta, \varphi)$.

In addition, the $2 \mathrm{D}$ reconstruction formula over a circular scan can be referred to as the solution of ultrasonic reflectivity imaging for a $2 \mathrm{D}$ reflecting medium. ${ }^{84}$

b. Planar geometry. Based on the mathematical techniques for ultrasonic reflectivity imaging, ${ }^{83} \mathrm{Xu}$ et al. ${ }^{85} \mathrm{de}-$ rived an exact Fourier-domain reconstruction formula for planar geometry. Köstli et al. ${ }^{86,87}$ presented a similar formula.

We assume that the measurement surface lies in the $z$ $=0$ plane, i.e., $\mathbf{r}_{0}=\left(x_{0}, y_{0}, 0\right)$ in a Cartesian coordinate system $\mathbf{r}=(x, y, z)[$ Fig. 6(b)]. The sample with a finite size lies above the plane, i.e., $p_{0}(\mathbf{r})=p_{0}(x, y, z)$ where $z>0$, and $p_{0}(\mathbf{r})=0$, otherwise. The exact reconstruction formula for $p_{0}(\mathbf{r})$ can be written as ${ }^{77,85}$

$$
\begin{aligned}
p_{0}(\mathbf{r})= & \frac{1}{4 \pi^{3}} \iint_{-\infty}^{+\infty} d x_{0} d y_{0} \int_{-\infty}^{+\infty} d k \tilde{p}_{d}\left(\mathbf{r}_{0}, k\right) \\
& \times \iint_{\rho=0}^{\rho=|k|} d u d v \exp [ \\
& \left.-i z \operatorname{sgn}(k) \sqrt{k^{2}-\rho^{2}}\right] \exp \left[i u\left(x_{0}-x\right)+i v\left(y_{0}-y\right)\right],
\end{aligned}
$$

where $\rho=\sqrt{u^{2}+v^{2}}, \operatorname{sgn}(k)=1$ when $k>0$, and $\operatorname{sgn}(k)=-1$ when $k<0$. If the source $p_{0}(\mathbf{r})$ and the measurement $\tilde{p}_{d}\left(\mathbf{r}_{0}, k\right)$ are expanded in the Fourier domain as

$$
\begin{aligned}
p_{0}(x, y, z)= & \frac{1}{(2 \pi)^{3}} \iiint \tilde{p}_{0}(u, v, w) \exp (-i u x-i v y \\
& -i w z) d u d v d w
\end{aligned}
$$

and

$$
\begin{aligned}
\tilde{p}_{d}\left(x_{0}, y_{0}, k\right)= & \frac{1}{(2 \pi)^{2}} \iint_{-\infty}^{+\infty} \widetilde{q}(u, v, k) \exp \left(-i u x_{0}\right. \\
& \left.-i v y_{0}\right) d u d v,
\end{aligned}
$$

then the relationship between the source distribution and the measured data can be expressed by

$$
\tilde{p}_{0}(u, v, w)=\frac{2 w \operatorname{sgn}(w)}{\sqrt{u^{2}+v^{2}+w^{2}}} \widetilde{q}\left(u, v, \operatorname{sgn}(w) \sqrt{u^{2}+v^{2}+w^{2}}\right) .
$$

Implementing the fast Fourier transform (FFT) accelerates the reconstruction computation. The reconstruction follows in three steps: (1) take the 2D FFT of $\tilde{p}_{d}\left(x_{0}, y_{0}, k\right)$ [inverse of Eq. (22)] to find the Fourier decomposition $\widetilde{q}(u, v, k)$ of $u$ and $v$ as a function of $k,(2)$ compute $\tilde{p}_{0}(u, v, w)$ from $\widetilde{q}(u, v, k)$ based on Eq. (23), and (3) take the inverse FFT of $\tilde{p}_{0}(u, v, w)$, i.e., Eq. (21), to find the initial pressure $p_{0}(x, y, z)$.

In addition, the $2 \mathrm{D}$ reconstruction formula can be referred to as the diffraction tomography theory as described in a book by Kak and Slaney ${ }^{88}$ or as the solution for ultrasonic reflectivity imaging in the case of an omnidirectional source receiver translated in a straight line. ${ }^{89}$ 
c. Cylindrical geometry. Xu et al. ${ }^{90}$ derived an exact reconstruction formula for the cylindrical geometry. This formula is much simpler and more stable than the reconstruction method reported for ultrasonic reflectivity imaging with a cylindrical scanning surface. ${ }^{83}$

As shown in Fig. 6(c), we assume that the measurement surface is a circular cylindrical surface $\mathbf{r}_{0}=\left(\rho_{0}, \varphi_{0}, z_{0}\right)$ in a circular cylindrical coordinate system $\mathbf{r}=(\rho, \varphi, z)$. The sample with a finite size lies within the cylinder, i.e., $p_{0}(\mathbf{r})$ $=p_{0}(\rho, \varphi, z)$ where $\rho<\rho_{0}$, and $p_{0}(\mathbf{r})=0$, otherwise. The exact reconstruction formula for $p_{0}(\mathbf{r})$ can be written as ${ }^{77}$

$$
\begin{aligned}
p_{0}(\mathbf{r})= & \frac{1}{2 \pi^{3}} \int_{0}^{2 \pi} d \varphi_{0} \int_{-\infty}^{+\infty} d z_{0} \int_{0}^{+\infty} d k \tilde{p}_{d}\left(\mathbf{r}_{0}, k\right) \\
& \times \int_{-k}^{+k} d \gamma \exp \left[i \gamma\left(z_{0}-z\right)\right] \sum_{n=-\infty}^{+\infty} \exp \left[\operatorname { i n } \left(\varphi_{0}\right.\right. \\
& -\varphi)] \frac{J_{n}\left(\rho \sqrt{k^{2}-\gamma^{2}}\right)}{H_{n}^{(1)}\left(\rho_{0} \sqrt{k^{2}-\gamma^{2}}\right)}
\end{aligned}
$$

where $J_{n}(\cdot)$ and $H_{n}^{(1)}(\cdot)$ are the Bessel function of the first kind and the Hankel function of the first kind, respectively. If the source $p_{0}(\mathbf{r})$ and the measurement $\tilde{p}\left(\mathbf{r}_{0}, k\right)$ are expanded in circular harmonics as

$$
\begin{aligned}
p_{0}(\rho, \varphi, z)= & \frac{1}{2 \pi} \int_{-\infty}^{+\infty} d \gamma \exp (-i \gamma z) \frac{1}{2 \pi} \sum_{n=-\infty}^{+\infty} \exp (-i n \varphi) \\
& \times \frac{(-i)^{n}}{2 \pi} \int_{0}^{+\infty} \mu d \mu J_{n}(\rho \mu) \widetilde{p}_{0 n}(\mu, \gamma),
\end{aligned}
$$

and

$$
\begin{aligned}
\tilde{p}_{d}\left(\varphi_{0}, z_{0}, k\right)= & \frac{1}{2 \pi} \int_{-\infty}^{+\infty} d \gamma \exp \left(-i \gamma z_{0}\right) \frac{1}{2 \pi} \sum_{n=-\infty}^{+\infty} \exp ( \\
& \left.-i n \varphi_{0}\right) \tilde{q}_{n}(\gamma, k),
\end{aligned}
$$

then the relationship between the source distribution and the measured data can be expressed by

$$
\widetilde{p}_{0 n}(\mu, \gamma)=\frac{4(+i)^{n} \widetilde{q}_{n}\left(\gamma, \sqrt{\mu^{2}+\gamma^{2}}\right)}{\sqrt{\mu^{2}+\gamma^{2}} H_{n}^{(1)}\left(\mu \rho_{0}\right)} .
$$

The reconstruction process follows in three steps: (1) take the 2D FFT of $\tilde{p}_{d}\left(\varphi_{0}, z_{0}, k\right)$ [inverse of Eq. (26)] to find the Fourier decomposition $\widetilde{q}_{n}(\gamma, k)$ of $n$ and $\gamma$ as a function of $k$, (2) compute $\widetilde{p}_{0 n}(\mu, \gamma)$ from $\widetilde{q}_{n}(\gamma, k)$ based on Eq. (27), and (3) take the Hankel transform over $\mu$ of $\tilde{p}_{0 n}(\mu, \gamma)$ and then inverse 2D FFT, i.e., Eq. (25), to find the initial pressure $p_{0}(\rho, \varphi, z)$.

In addition, Norton and Vo-Dinh ${ }^{91}$ presented a 2.5dimensional (2.5D) (i.e., homogeneous along the $z$ axis) reconstruction algorithm, which actually can be obtained directly by simplifying the 3D solution of Eq. (24).

\section{Time-domain algorithms}

In Sec. VI C 1, we introduced approximate time-domain algorithms, such as the Radon transform approximation formula Eq. (11) and the modified back-projection formula Eq. (12). Following these algorithms, Finch et al. ${ }^{92}$ reported on a time-domain reconstruction formula for the spherical geometry based on the inverse of the spherical Radon transform. Based on Eq. (9), the formula takes the following form as

$$
p_{0}(\mathbf{r})=-\frac{1}{2 \pi r_{0}} \nabla^{2} \int_{S_{0}} d S_{0} \frac{\Psi\left(\mathbf{r}_{0}, \bar{t}=\left|\mathbf{r}-\mathbf{r}_{0}\right|\right)}{\left|\mathbf{r}-\mathbf{r}_{0}\right|},
$$

where $\Psi\left(\mathbf{r}_{0}, \bar{t}\right)=\bar{t} \int_{0}^{\bar{t}} p_{d}\left(\mathbf{r}_{0}, \bar{t}\right) d \bar{t}$ and $\nabla$ is the gradient over variable $\mathbf{r}$. The reconstruction first back-projects the data $\Psi\left(\mathbf{r}_{0}, \bar{t}\right)$ to the image space and then takes the space filtering by $\nabla^{2}$. Both Eqs. (16) and (28) are exact inverse solutions. In addition, if we introduce velocity potential $\Phi(\mathbf{r}, \bar{t})$ defined by $\Phi(\mathbf{r}, \bar{t})=-\int_{0}^{\bar{t}} p(\mathbf{r}, \bar{t}) d \bar{t} / \rho c$ ( $\rho$, the density), ${ }^{59}$ we can rewrite Eq. (28) as

$$
\Phi_{0}(\mathbf{r})=-\frac{1}{2 \pi r_{0}} \nabla^{2} \int_{S_{0}} d S_{0} \Phi_{d}\left(\mathbf{r}_{0}, \bar{t}=\left|\mathbf{r}-\mathbf{r}_{0}\right|\right),
$$

where $\Phi_{0}(\mathbf{r})=-p_{0}(\mathbf{r}) / \rho c$ and $\Phi_{d}\left(\mathbf{r}_{0}, \bar{t}\right)=-\int_{0}^{\bar{t}} p_{d}\left(\mathbf{r}_{0}, \bar{t}\right) d \bar{t} / \rho c$. $\Phi(\mathbf{r}, \bar{t})$ are smooth functions that can significantly depress noise.

In a study of exact Fourier-domain reconstructions, $\mathrm{Xu}$ and Wang ${ }^{93}$ derived a universal back-projection formula for all three types of imaging geometries: planar, spherical, and cylindrical surfaces as follows:

$$
p_{0}(\mathbf{r})=-\frac{2}{\Omega_{0}} \nabla \cdot \int_{S_{0}} \mathbf{n}_{0}^{s} d S_{0}\left[\frac{p_{d}\left(\mathbf{r}_{0}, \bar{t}\right)}{\bar{t}}\right]_{\bar{t}=\left|\mathbf{r}-\mathbf{r}_{0}\right|},
$$

where $\Omega_{0}$ is the solid angle of the whole measurement surface $S_{0}$ with respect to the reconstruction point inside $S_{0}: \Omega_{0}=2 \pi$ for the planar geometry and $\Omega_{0}=4 \pi$ for the spherical and cylindrical geometries. The inversion formula Eq. (28) for the spherical geometry given by Finch et al. ${ }^{92}$ can be simplified to Eq. (30) (See Ref. 93 for details).

Further, Eq. (30) can be rewritten in a back-projection form as

$$
p_{0}(\mathbf{r})=\int_{\Omega_{0}} b\left(\mathbf{r}_{0}, \bar{t}=\left|\mathbf{r}-\mathbf{r}_{0}\right|\right) d \Omega_{0} / \Omega_{0},
$$

with the back-projection term related to the measurement at position $\mathbf{r}_{0}$,

$$
b\left(\mathbf{r}_{0}, \bar{t}\right)=2 p_{d}\left(\mathbf{r}_{0}, \bar{t}\right)-2 \bar{t} \partial p_{d}\left(\mathbf{r}_{0}, \bar{t}\right) / \partial \bar{t},
$$

where $d \Omega_{0}=d S_{0} /\left|\mathbf{r}-\mathbf{r}_{0}\right|^{2} \cdot\left[\mathbf{n}_{0}^{s} \cdot\left(\mathbf{r}-\mathbf{r}_{0}\right) /\left|\mathbf{r}-\mathbf{r}_{0}\right|\right]$, and the unit vector $\mathbf{n}_{0}^{s}$ is the normal of the measurement surface pointing to the source. The element $d \Omega_{0}$ is the solid angle of the small element $d S_{0}$ with respect to the point $P$ of the reconstruction. Actually, the ratio $d \Omega_{0} / \iint d \Omega_{0}$ is the solid-angle weighting factor, which stands for the contribution from the detection element $d S_{0}$ to the reconstruction at point $P$. The factor $\left[\mathbf{n}_{0}^{s} \cdot\left(\mathbf{r}-\mathbf{r}_{0}\right) /\left|\mathbf{r}-\mathbf{r}_{0}\right|\right]$ is the angle between the normal of $d S_{0}$ and $\mathbf{r}-\mathbf{r}_{0}$ (the vector pointing from the point of detection to the point of the reconstructed source). The factor $\bar{t}=\left|\mathbf{r}-\mathbf{r}_{0}\right|$ compensates for the acoustic wave diffraction attenuation that is inversely proportional to the traversing distance from the acoustic source to the detection element. Obviously, the modified back-projection Eq. (12) is an approximation of Eq. (31) under the following conditions: (1) $2 p_{d}\left(\mathbf{r}_{0}, \bar{t}\right) / \bar{t}$ is negligible as $|k|\left|\mathbf{r}-\mathbf{r}_{0}\right| \gg 1$, and (2) $\left[\mathbf{n}_{0}^{s} \cdot\left(\mathbf{r}-\mathbf{r}_{0}\right) /\left|\mathbf{r}_{0}-\mathbf{r}\right|\right] \approx\left[\mathbf{n}_{0}^{s}\right.$ 


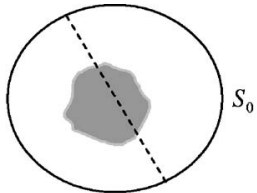

(a)

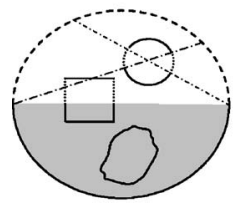

(c)

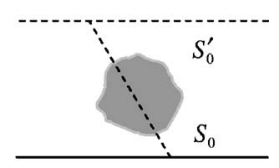

(b)

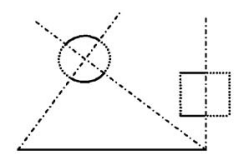

(d)
FIG. 7. Measurement surface enclosing: (a) and (b). Diagram of detection region: (c) and (d).

$\left.\cdot\left(-\mathbf{n}_{0}\right)\right]$ as $|\mathbf{r}| \ll\left|\mathbf{r}_{0}\right|$, i.e., the source is located near the center region. In reality, the measured PA signals may have some amplitude or phase distortions caused by the temporal response of the detection system, including the illumination pulse or the pulse response of the detector. If the system response can be known, a deconvolution method can be used to minimize these distortions and recover $p_{d}\left(\mathbf{r}_{0}, \bar{t}\right)$ or $\partial p_{d}\left(\mathbf{r}_{0}, \vec{t}\right) / \partial t$. A discussion about how to implement the backprojection algorithm can be found in Ref. 93.

In addition, $\mathrm{Xu}$ and Wang ${ }^{94}$ applied the time reversal method to PAT and TAT with a diffracting source using only the field, rather than both the field and its gradient, measured on an arbitrary closed surface that enclosed the initial source. They presented a formal back-projection solution with the expression of Green's function subject to the homogeneous Dirichlet boundary condition. However, it is usually difficult to find an analytic expression for an arbitrary boundary. Therefore, under the ray approach (a geometrical optics approximation that ignores the multiple reflections from the Dirichlet boundary), they derived an approximation formula that is identical to Eq. (31) in the full view case. This result actually indicates that, in the spherical geometry, the multiple reflections from the Dirichlet boundary cancel out in the end. See Ref. 93 for detailed proof.

\section{Aperture enclosing and limited view}

In exact algorithms, the PA sources should be detectable in a full view by a closed spherical surface, a planar surface of an infinite extent, or a cylindrical surface of an infinite length. In other words, as shown in Figs. 7(a) and 7(b), each point of the object can be detected by the detector's trajectory with $4 \pi$ steradians in the $3 \mathrm{D}$ enclosing case (sphere and cylinder) and with $2 \pi$ radians in the $2 \mathrm{D}$ enclosing case (circle); $2 \pi$ steradians are required in the planar surface and $\pi$ radians for a line measurement.

Actually, we can regard the planar geometry as a special enclosing case if we assume that at $\mathbf{r}_{0}^{\prime}$ there is another measurement surface $S_{0}^{\prime}$ that is parallel to $S_{0}$ and that these can be combined to provide a $4 \pi$ steradian enclosure, as shown in Fig. 7(b). However, since $S_{0}^{\prime}$ is far away from the finitesize object, the measurement $p_{d}\left(\mathbf{r}_{0}^{\prime}, t\right)$ over $S_{0}^{\prime}$ alone is sufficient to provide an exact reconstruction in the limits of the Radon transform $\left(\mathbf{r}_{0}^{\prime} \rightarrow \infty\right)$, as discussed above in Sec. VI C 1. Therefore, it is "reasonable" that the measurement $p_{d}\left(\mathbf{r}_{0}, t\right)$ over $S_{0}$ alone offers an exact reconstruction. Likewise, the line measurement in the $2 \mathrm{D}$ case with a $\pi$ radian "enclosure" is sufficient for an exact reconstruction.

However, in practical applications, the measurement surfaces are generally finite and partially closed, and the PA signals cannot be collected from all directions. For example, the solid angle of detection is at most $2 \pi$ steardians for a breast in a hemispherical form. Therefore, what we face in many actual cases is an incomplete data problem. Algorithms for full-view data can be extended to the limited-view case simply by assuming that the unmeasured data are zero or by estimating them from measured data through other methods, such as interpolation. In practical implementations, limitedview problems usually result in the loss of some part of the high-frequency information and, hence, the blurring of some sharp details. ${ }^{69,95}$

Both Xu et al. ${ }^{69}$ and Pan et al. ${ }^{95}$ have conducted numerical simulations in 2D circular measurement cases. Their results indicate that for many objects possessing boundaries, the images reconstructed from reduced-scan data, such as the $\pi$-scheme data, can have a numerical accuracy that is similar to that of full-scan images. Similar results hold in the 3D case.

Patch $^{96}$ presented a study of the partial scan problem in TAT using ultrasound transducers located on the bottom of a spherical bowl, where $\{z<0\}$. The inversion formulas for the "complete data" case, where the transducers measured all over the bowl, weight the data from the lower hemisphere more heavily as reconstruction points in $\{z<0\}$. The unmeasured data, corresponding to transducer locations on the top of the bowl, could be estimated from the measured data based on the consistency conditions of the data; however, this process is clearly unstable, but somewhat tempered by the reconstruction's $1 / r$ weighting.

A "detection region," within which all points have sufficient detection views, can be defined by the following rule: ${ }^{69}$ All lines connecting nondetection points along the scanning circle in $2 \mathrm{D}$ imaging (or a sphere in $3 \mathrm{D}$ imaging) cover the "invisible" domain and its complement is covered by all of the lines connecting the detection points from the detection region. In the invisible domain, some boundaries can be recovered stably while others blur away. Namely, the parts of the boundaries that allow normal lines to pass through a detector position, and only those, can be stably recovered. The above conclusions are illustrated in Figs. 7(c) and 7(d), where the invisible parts of the object boundaries, i.e., the ones that will be blurred during the reconstructions, are shown with dotted lines and the detection region is shaded.

Particularly, the scanning view is quite limited in the measurement of only a part of a line or a plane; consequently, artifacts and interface blurring appear in the reconstructed images. In fact, one can never have an object immersed entirely into the detection region in the planar and linear detection geometries because the normal lines to any interfaces that are orthogonal to the detector plane (line) never pass through a detector. Consequently, those parts of the interfaces will be blurred in any kind of reconstruction. 
To reduce the artifacts, we can combine multiplanar measurements by including, for example, an open-box-shaped measurement surface.

In the reconstruction, view-angle weighting can help to minimize a particular distortion as described below. For example, when imaging a human breast with a hemispherical measurement surface, the solid angle for all detectors on the hemispherical surface with respect to a location inside the breast is less than $4 \pi$ and varies at different locations. Thus, for sources at different locations but with the same amplitudes, the amplitudes in the reconstruction image will vary at different locations as well, which causes distortion in reconstruction. A straightforward way to compensate for this kind of reconstruction distortion, which results from a limited view, is to normalize the reconstruction at each location by a total solid angle weight $\iint \mathrm{d} \Omega_{0}$, as shown in Eq. (31).

Half-closed ( $2 \pi$ steradians for 3D and $\pi$ radians for 2D) measurement can provide a "reasonable" reconstruction. The measured data become more "Radon-like" as the integration shells (arcs) approximate planes (lines) when the detectors move away from the objects; therefore, more accurate images can be reconstructed from a half-enclosing scan.

\section{Spatial resolution}

Spatial resolution, one of the most important parameters in imaging, is limited in PAT by many factors. The aforementioned reconstruction model is based on the following assumptions: (1) homogeneous sound speed, (2) full-angle view, (3) impulse excitation, (4) wideband detection, (5) point detector measurement, and (6) continuous sampling. Sometimes, these assumptions may not be realistic. For example, acoustic inhomogeneity blurs a reconstructed image because the resulting sound speed variations may cause a significant change in the time of flight for sound to travel from the source to detectors. In practice, we may slightly adjust the time of flight in the reconstruction to obtain a good "focused" image of the region of interest. As discussed previously, a limited-angle view also affects spatial resolution due to gaps in the raw data. In the investigation of an object of a size larger than the micron scale in soft tissue, the thermal diffusion effect on the PA signal excited by a pulse with a duration of less than the micron scale is negligible, i.e., the thermal confinement condition is met. However, the stress propagation in the pulse duration can significantly blur the PA signal as expressed by a convolution in Eq. (7), i.e., the stress confinement condition is not met. This blurring effect actually is due to the bandwidth of the PA signal that is determined by the finite width of the excitation pulse.

In reality, any ultrasound detector has a finite sensing aperture, rather than a point, which results in a finite spatialfrequency bandwidth, and any detection system (including an ultrasound detector) has a finite response time, which results in a finite temporal-frequency bandwidth. If the detection system is linear and time invariant, the real signal detected at position $\mathbf{r}_{0}$ can be expressed by the convolution of the surface integral over the aperture of the detector over the impulse response $I_{d}(t)$ of the detection system,

$$
p_{d}^{\prime}\left(\mathbf{r}_{0}, t\right)=\int_{-\infty}^{+\infty} d \tau I_{d}(t-\tau) \iint_{\mathbf{r}^{\prime}} d^{2} \mathbf{r}^{\prime} W\left(\mathbf{r}^{\prime}\right) p_{e}\left(\mathbf{r}_{0}+\mathbf{r}^{\prime}, \tau\right),
$$

where $\mathbf{r}^{\prime}$ points to an element on the surface of the detector with respect to the position of the detector $\mathbf{r}_{0}, W\left(\mathbf{r}^{\prime}\right)$ is a weighting factor that represents the contribution from the various surface elements of the detector to the total signal received by the detector, and $p_{e}\left(\mathbf{r}_{0}, t\right)$ is expressed in Eq. (7). The measured data $p_{d}^{\prime}\left(\mathbf{r}_{0}, t\right)$ are used to reconstruct the initial pressure $p_{0}(\mathbf{r})$. We combine the excitation pulse with the PA detection system and denote the temporal impulse response of the detection system as $H(t)$ that equals

$$
H(t)=\int_{-\infty}^{+\infty} d \tau I_{d}(t-\tau) I_{e}(t) .
$$

Then, one can rewrite Eq. (33) in the Fourier domain as

$$
\widetilde{p}_{d}^{\prime}\left(\mathbf{r}_{0}, k\right)=\widetilde{H}(k) \iint_{\mathbf{r}^{\prime}} d^{2} \mathbf{r}^{\prime} W\left(\mathbf{r}^{\prime}\right) \widetilde{p}\left(\mathbf{r}_{0}+\mathbf{r}^{\prime}, k\right),
$$

with $\widetilde{H}(k)$ being the Fourier transform of $H(t)$ and

$$
\widetilde{p}_{d}\left(\mathbf{r}_{0}, k\right)=-i k \iiint d^{3} \mathbf{r} p_{0}(\mathbf{r}) \widetilde{G}_{k}\left(\mathbf{r}, \mathbf{r}_{0}\right) .
$$

The pressure $\widetilde{p}_{d}\left(\mathbf{r}_{0}, k\right)$ is the idealized measurement with a point detector $\left[W\left(\mathbf{r}^{\prime}\right)=\delta\left(\mathbf{r}^{\prime}\right)\right]$ and a Dirac-delta impulse excitation and detection $[H(t)=\delta(t)]$. As mentioned before, $p_{0}(\mathbf{r})$ can be perfectly recovered from the idealized measurement $\tilde{p}_{d}\left(\mathbf{r}_{0}, k\right)$.

A point-spread function (PSF) can be used to describe spatial resolution. PSFs in the full-angle view, as a function of the bandwidth of the measurement system and the finite size of the detector aperture, have been investigated for the spherical, planar, and cylindrical detection surfaces. ${ }^{97,98} \mathrm{Be}-$ low, we summarize the results as well as discuss the discrete spatial sampling effect on reconstruction.

\section{Bandwidth}

As shown in Eq. (35), the temporal-frequency bandwidth of a detection system determines the temporal-frequency bandwidth of the detected PA waves. The resolution of a PA image is actually limited by the bandwidth of the detected PA waves. If we set the zero time point at the center of the impulse response of the detection system $H(t)$, then $H(t)$ is assumed to be split into even and odd portions as $E(t)$ and $O(t)$, respectively; and, further, the corresponding PSF is found to $\mathrm{be}^{97}$

$$
\operatorname{PSF}(R)=-\left(\frac{1}{2 \pi R}\right)\left[\frac{d E(R)}{d R}\right],
$$

where $R$ is the distance between the point of observation and the acoustic source. The odd part is canceled out in the reconstruction.

We take the rectangle-shaped bandwidth, which is cut off at the frequency $f_{c}$, as an example. In this case, the impulse response function $H(t)$ is the sinc function, $\operatorname{sinc}(K t) K / \pi$, where $\operatorname{sinc}(x)=\sin (x) / x$ and $K=2 \pi / \lambda_{c}\left(\lambda_{c}\right.$, the cutoff wavelength $=2 \pi c / f_{c}$ ). We find that the dependence of 
the PSFs on the bandwidth of all of the measurement geometries shares the same space-invariant expression as ${ }^{97}$

$$
\operatorname{PSF}(R)=\left(\frac{K^{3}}{2 \pi^{2}}\right)\left[\frac{j_{1}(K R)}{K R}\right],
$$

where $j_{1}(\cdot)$ is a first-order spherical Bessel function of the first kind. If the full width at half maximum (FWHM) of the PSF is used to represent the spatial resolution, then, in this case, $R_{H} \approx 0.80 \lambda_{c}$. In an analogy to the Rayleigh criterion, an alternative definition of spatial resolution is the distance between two points in the object space when the maximum (positive) of the first PSF overlaps with the first minimum (negative) of the second. By this definition, the spatial resolution becomes $R_{R} \approx 0.92 \lambda_{c}$, which is slightly wider than the FWHM definition. The Rayleigh criterion is more appropriate, as negative-value artifacts are introduced into the reconstruction due to the band limit. Note that this temporalfrequency bandwidth-limited resolution is isotropic around the point of observation.

\section{Sensing aperture}

As shown in Eq. (35), the finite sensing aperture of the transducer, or element, in an array acts as a spatial filter along the measurement surface; this actually determines the spatial-frequency bandwidth of the detected PA waves. Hence, the finite sensing aperture blurs the resolution of the PAT image, but the dependence of the PSF on the aperture size differs among the various measurement configurations. ${ }^{97}$ Rigorous expressions can be given when the detector surfaces are assumed to have the same curvature properties as the measurement geometries and the temporal bandwidth is unlimited. Of course, the detector aperture is much smaller than the measurement surface. The axial extension of the PSF due to the detector aperture is negligible compared to the lateral extension.

If the detection scan is along a straight line, such as a planar scan or a $z$ scan in the cylindrical scan, the lateral extension of the PSF approximates $R_{L}(h) \approx \delta,{ }^{97}$ where $h$ is the distance of the point source to the scan line and $\delta$ is the diameter of the detector. Hence, the lateral resolution in this case is blurred by the detector aperture no matter where the point source is.

If the detection scan is along a circle, such as a spherical or cylindrical scan, the lateral extension of the PSF can be estimated by $R_{C}(r) \approx\left(r / r_{0}\right) \delta,{ }^{97,98}$ where $r$ is the distance between the point source and the center of the scan circle, $r_{0}$ is the radius of the scan circle, and $\delta$ is the diameter of the detector. The farther the point source is away from the center, the wider the PSF is and vice versa. Therefore, the lateral resolution improves when the point source approaches the center of the scan. This is because the scan along a circle actually measures the angular information with a resolution of $\delta / r_{0}$.

\section{Discrete sampling}

Discrete sampling in the measurement will also affect the resolution of a PAT image. The PA signals are sampled around the source at a series of discrete spatial detection positions with a spatial sampling period (the inverse of the frequency), and at each detection position the signals are sampled at a series of discrete temporal points with a temporal sampling period. According to the sampling (Nyquist) theorem, to reconstruct a signal from its periodically sampled version, the sampling frequency must be at least twice the maximum frequency of the signal, which is applicable to both spatial and temporal samplings. Otherwise, aliasing artifacts occur and cause confusion and serious measurement errors, in which frequency information that is higher than half the sampling frequency will disguise itself as lowfrequency information. From a user's point of view, aliasing limits the useful frequency range such that the highest frequency component is no more than half the sampling frequency, which, consequently, limits the spatial resolution of the reconstruction.

If the spatial sampling period is less than half the diameter of the sensing aperture of the detector, the reconstruction can avoid, or significantly reduce, aliasing due to the spatially discrete sampling. However, a further decrease in the spatial sampling period does not improve the spatial resolution of the images nor provide other significant advantages. It does, however, cause an increase in the amount of raw data. In practice, it is reasonable to set the discrete spatial sampling period to be two to five times smaller than the sensing aperture of the detector in a scanning system. Then, the sensing aperture size of the detector limits the lateral resolution while the temporal-frequency bandwidth affects both the axial and lateral resolutions.

\section{E. Large planar detector}

For simplicity, assuming that $W\left(\mathbf{r}^{\prime}\right)=1$ and a $\delta(t)$ EM source is used, we can simplify Eq. (32) to

$$
p_{s}\left(\mathbf{r}_{0}, t\right)=\iint_{\mathbf{r}^{\prime}} d^{2} \mathbf{r}^{\prime} p_{d}\left(\mathbf{r}_{0}+\mathbf{r}^{\prime}, t\right)
$$

where the subscript $s$ denotes the measurement is a surface integral. Substituting Eq. (9) into Eq. (39),

$$
p_{s}\left(\mathbf{r}_{0}, t\right)=\iint_{\mathbf{r}^{\prime}} d^{2} \mathbf{r}^{\prime} \frac{\partial}{\partial t}\left[\frac{t}{4 \pi} \iint_{\left|\mathbf{r}_{0}+\mathbf{r}^{\prime}-\mathbf{r}\right|=c t} p_{0}(\mathbf{r}) d \Omega\right] .
$$

Now, the problem is to reconstruct $p_{0}(\mathbf{r})$ from $p_{s}\left(\mathbf{r}_{0}, t\right)$ based on Eq. (40). It will be mathematically difficult if the detector has a random-shaped aperture. However, in special cases, such as with a planar-surface detector, Eq. (40) can be further simplified. Taking the Fourier transform of Eq. (40), we have

$$
p_{s}\left(\mathbf{r}_{0}, k\right)=-i k \iiint d^{3} r p_{0}(\mathbf{r}) \iint_{\mathbf{r}^{\prime}} d^{2} \mathbf{r}^{\prime} \widetilde{G}_{k}\left(\mathbf{r}, \mathbf{r}_{0}+\mathbf{r}^{\prime}\right) \text {. }
$$

As shown in Fig. 8, we assume the planar detector has a large surface, ideally infinite in size. The vector $\mathbf{r}_{0}$ is along the normal of the planar surface, and $\mathbf{r}^{\prime}$ in this case is perpendicular to $\mathbf{r}_{0}$. We use the local coordinate system aligned with $\quad \mathbf{r}_{0}, \quad$ in which $\quad \mathbf{r}_{0} \rightarrow(0,0,0), \quad \mathbf{r} \rightarrow\left(x_{l}, y_{l}, z_{l}\right), \quad \mathbf{r}^{\prime}$ $\rightarrow\left(x_{l}^{\prime}, y_{l}^{\prime}, 0\right)$, and $\iint_{\mathbf{r}^{\prime}} d^{2} \mathbf{r}^{\prime}=\iint_{s} d x_{l}^{\prime} d y_{l}^{\prime}$. Then, considering the expansion of Green's function in the Cartesian coordinates 


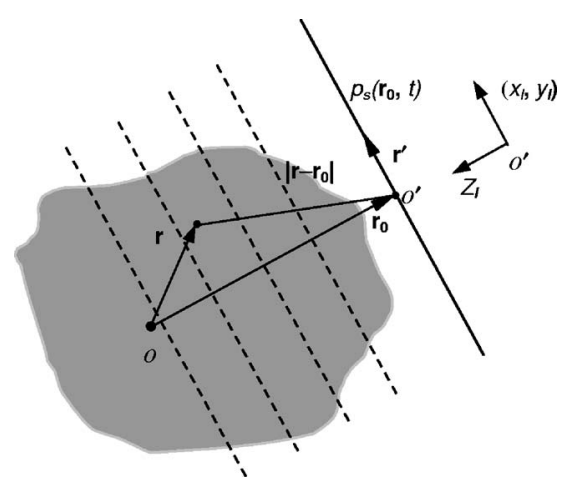

FIG. 8. Diagram of photoacoustic measurement at $\mathbf{r}_{0}$ with a large planar detector.

function (see, e.g., Ref. 97), we can simplify Eq. (41) to

$$
p_{s}(0, k)=\frac{1}{2} \iiint p_{0}\left(x_{l}^{\prime}, y_{l}^{\prime}, z_{l}^{\prime}\right) \exp \left(i k z_{l}^{\prime}\right) d x_{l}^{\prime} d y_{l}^{\prime} d z_{l}^{\prime} .
$$

Further, taking the inverse Fourier transform of Eq. (42), we get in the local coordinate

$$
2 p_{s}(0, \bar{t})=\iint p_{0}\left(x_{l}^{\prime}, y_{l}^{\prime}, z_{l}^{\prime}=\bar{t}\right) d x_{l}^{\prime} d y_{l}^{\prime} .
$$

The above equation indicates that the PA signal detected by a planar surface at a given time actually measures the integral of the initial PA source, or energy density function, over a planar surface parallel to the detector surface at a "delayed" distance characterized by the acoustic time of flight between these two planar surfaces. Therefore, the standard Radon transform can be directly applied to the measured data that are detected through rotating the planar detector tangentially to a spherical surface of the object under study. Thus, if we assume that the detector scans over a spherical surface with a radius of $r_{0}$, the reconstruction formula can be written as

$$
p_{0}(\mathbf{r})=-\frac{1}{4 \pi^{2}} \iint_{\Omega_{0}} \frac{\partial^{2}}{\partial \vec{t}^{2}} p_{s}\left(\mathbf{r}_{0}, \bar{t}=r_{0}-\frac{\mathbf{r}_{0} \cdot \mathbf{r}}{r_{0}}\right) d \Omega_{0},
$$

where $\Omega_{0}$ denotes the detection spherical surface and covers $4 \pi$ steradians and $\mathbf{r}_{0}$ is along the radius of the detection spherical surface and perpendicular to the detector's surface. In addition, replacing 4 by 2 in Eq. (44) gives the reconstruction formula with the measured data only on half of the detection spherical surface since a $2 \pi$-steradian measurement is sufficient in the Radon transform. A mathematical proof can be found in Ref. 58. Similarly, if the sample is from a homogenous distribution along the $z$ axis, a circular scan around the cylinder enclosing the sample is sufficient for the reconstruction based on the 2D Radon transform.

Spatial resolution using a planar receiver is determined by the temporal frequency bandwidth, since the finite size of the receiver is explicitly included in the measurement and reconstruction. Spatial resolution is space invariant and highresolution imaging should be possible with a high-bandwidth receiver. However, it is not practical to make a transducer array using multiple large planar receivers and, therefore, we have to rotate a large receiver to cover the entire measurement surface. By contrast, as discussed in the previous sections, small receivers, used for simulating point measurement data, introduce some blurring effects in the reconstruction with analytical inversion formulas. However, a transducer array with multiple small elements can speed the data acquisition.

\section{EXPERIMENTS}

\section{A. Breast imaging and cancer detection}

Breast cancer remains a leading cause of death among women in many parts of the world. However, better diagnosis and treatment have noticeably improved the outcomes for women contracting this disease. Even though we now know that the earlier treatment begins, the greater the chances for survival, the current technologies for breast cancer detection are not particularly well suited for identifying small and/or deep tumors in the human breast. Film-screen x-ray mammography is still the gold standard of breast imaging technology, although it may miss up to $20 \%$ of existing lesions while at the same time identifying many false positives. According to the U.S. Institute of Medicine, ${ }^{99}$ the variety and sophistication of alternative imaging technologies under development have increased greatly. These methods can be divided into three general categories: (1) those that are currently in use, such as x-ray mammography and ultrasound, and are being further refined; (2) those that are commonly used for medical imaging, such as magnetic resonance imaging (MRI), but that are still experimental with regard to breast cancer detection; and (3) novel imaging modalities that may be used in the future.

PAT, or TAT, belongs to the third imaging category for breast cancer detection. The following advantages make PAT stand out among the numerous new imaging modalities: (1) PAT uses nonionizing radiation and, therefore, is harmless to humans (unlike x-ray mammography); (2) PAT relies on rf or light absorption that is sensitive to tissue abnormality, and hence it has the potential for early cancer detection; (3) PAT can provide submillimeter spatial resolution with the detection of megahertz ultrasound exited by short $\mathrm{rf}$ or laser pulses; (4) PAT does not require breast compression and, therefore, is less painful than x-ray mammography; (5) PAT can potentially be applied in vivo for real-time imaging with the implementation of an ultrasound detection array, and the same array can also produce conventional ultrasound images for comparison and codisplay of two types of contrast; (6) a PAT system is relatively inexpensive (comparable to the ultrasound system plus a rf or laser source); (7) a PAT system can also easily incorporate other rf or optical detection systems to produce pure if or optical images so that different types of contrast in the same imaging region can be codisplayed to provide more information for diagnostics; and (8) PAT is particularly well adapted for use with the physical properties of the human breast. Human breast consists of soft tissues with a speed variation in the range of $10 \%,{ }^{18}$ in which ultrasound propagation distortion is tolerable. ${ }^{79}$ Breast tissue is also easily accessible to both EM energy delivery and 


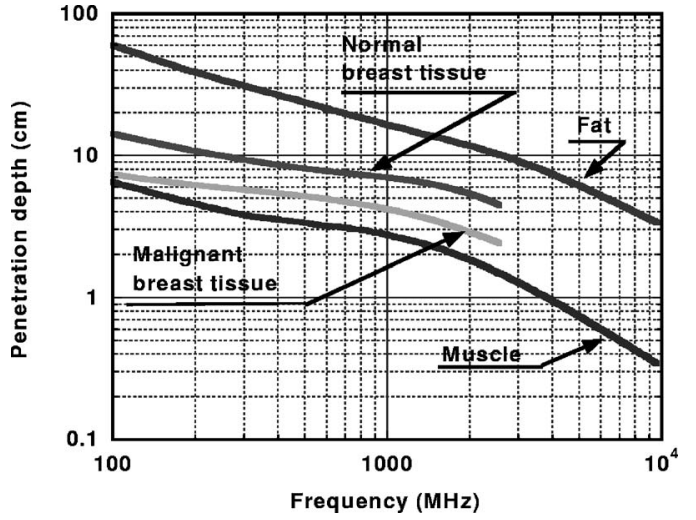

FIG. 9. rf penetration depths in various tissues.

ultrasound detection. Based on EM excitation sources, two types of PAT technologies for breast imaging are currently under development: rf-based TAT and laser-based PAT.

\section{1. rf-based imaging}

Cancer tissue is more rf absorbant than the surrounding normal tissue due to its increased water or ionic concentration. ${ }^{100}$ Figure 9 shows a comparison of the EM penetration depth in different types of tissues, which was computed based on the data reported in Ref. 100. In that data, the rf contrast between malignant breast tissue and normal breast is about two times. It is possible that contrast agents may further improve rf absorption in a tumor region. However, so far, no research on rf absorption contrast agents in soft tissues has been reported.

Two groups have made significant contributions in the area of rf-induced ultrasound imaging for breast cancer detection. One group, under the leadership of Dr. Robert Kruger, has developed thermoacoustic computed tomography (TCT) scanners with $434 \mathrm{MHz}$ radio waves to image the breast. They have designed several different versions of TCT systems. ${ }^{101-104}$ At the beginning, they situated multiple single transducers on a spherical bowl to provide array detection. However, the calibration of a large number of transducers was difficult, so later they used an ultrasonic array with multiple elements. After tests with phantom samples, such as a lamb kidney ${ }^{65}$ or a porcine kidney, ${ }^{102}$ they moved to clinical testing. ${ }^{103,104}$ In this work, ${ }^{104}$ they were able to visualize quite well most of the cysts that could be seen on ultrasound images; however, fibroadenomas could not be visualized ex- cept in the case of a fibroadenoma with associated atypical hyperplasia. The group attributed the lack of visualization of cancers to inadequate imaging quality, etc. We believe the main problem in their system was that the rf absorption at $434 \mathrm{MHz}$ is too weak and the SNR was not high enough. Figure 10(a) shows a diagram of their recent setup (Patent 6633774). ${ }^{101}$ A triplanar array, consisting of a total of 128 rectangular elements, was designed to approximate a spherical surface with a diameter of $170 \mathrm{~mm}$. The distribution of the detector elements was chosen to provide approximately uniform coverage over the surface of a hemisphere when it is rotated to a plurality of angles about the vertical axis. Each element is $7 \mathrm{~mm}$ wide and $8 \mathrm{~mm}$ high and has a wide bandwidth with a center frequency of $1 \mathrm{MHz}$. rf pulses with a $0.7 \mu$ s duration were directed toward the breast via eight waveguides arrayed radially around the breast, which remained stationary. Figure 10(b) shows a TCT image of a normal breast. The field of view of the image is 20 $\times 20 \mathrm{~cm}^{2}$ and has a $0.78 \mathrm{~mm}$ thickness because each voxel is a $0.78 \mathrm{~mm}$ cube. Tissues with higher water concentrations (e.g., skin and glandular tissue) display greater rf absorption than tissues with lower water concentrations (e.g., fat).

Our own group developed a prototype of an imaging system utilizing $3 \mathrm{GHz}$ microwaves. ${ }^{73,76,85,90,98}$ rf absorption at this frequency is about twofold stronger than that at $434 \mathrm{MHz}$, according to the data shown in Fig. 9. Figure 11(a) shows a diagram of the circular measurement configuration in our initial study. ${ }^{98}$ An ultrasound transducer driven by a step motor scanned around the sample on a horizontal $x-y$ plane; this mechanical scanning will eventually be replaced by a circular transducer array. Both the transducer and the sample under study were immersed in a container filled with a coupling medium such as mineral oil. The microwave pulses transmitted from a $3 \mathrm{GHz}$ microwave generator had a pulse energy of $10 \mathrm{~mJ}$ and a pulse width of $0.5 \mu \mathrm{s}$. Microwave energy was delivered to the sample by a rectangular waveguide with a cross section of $72 \times 34 \mathrm{~mm}^{2}$ that was recently replaced by a waveguide horn to provide a larger illumination area. Figure 11(b) shows the image of a phantom sample with five gel absorbers (made of gelatin and water) buried in a fat base, which was acquired by a $2.25 \mathrm{MHz}$ unfocused ultrasonic transducer. The high contrast is due to high rf absorption in gel (high water content) and low rf absorption in fat (low water content). A preliminary study of breast cancer imaging was reported, ${ }^{105,106}$ in which excised

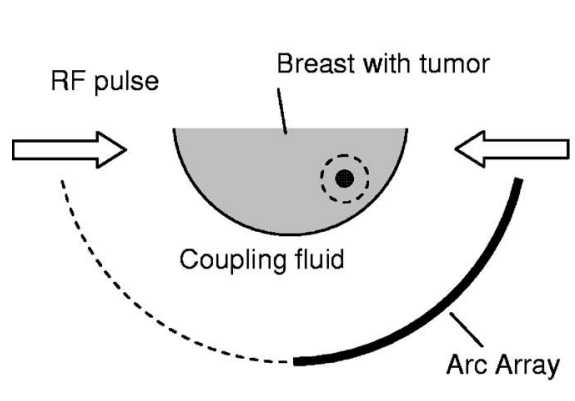

(a)

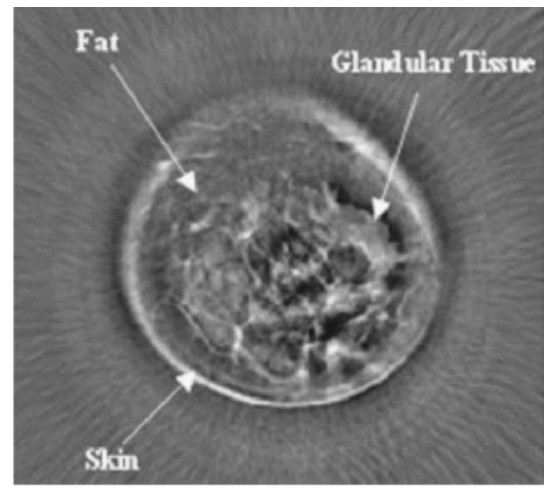

(b)
FIG. 10. (a) Side view schematic of a breast imager with spherical scans. (b) TCT image of a normal breast (Courtesy of OptoSonics, Inc.). 


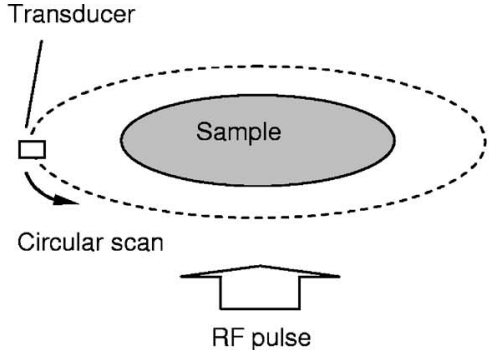

(a)

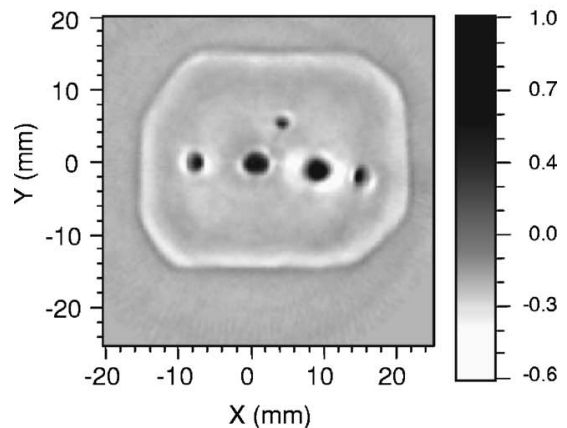

(b)

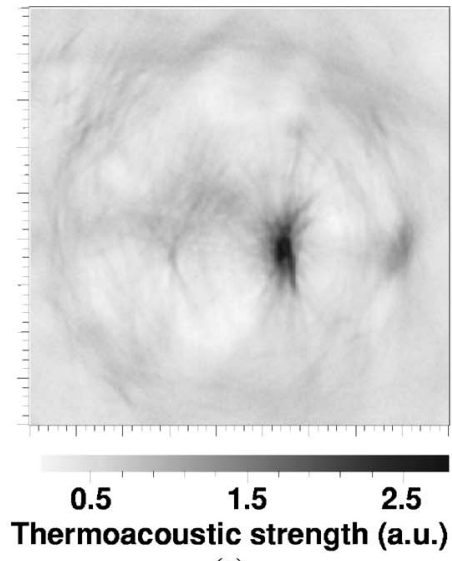

(c)

FIG. 11. (a) Diagram of circular scan. (b) Reconstructed thermoacoustic image. (c) TAT image of a breast mastectomy specimen (Ref. 106).

breast specimens were imaged and tumor regions were clearly located. An example of the TAT image of a mastectomy specimen, which was acquired by a $1 \mathrm{MHz}$ cylindrically focused ultrasonic transducer, is shown in Fig. 11(c). ${ }^{106}$

In summary, for breast imaging, the first challenge of TAT is the delivery of rf energy to the breast. Because of its long wavelength, rf energy does not readily focus into a small region of interest for the scanning rf focuses to generate images with good resolution. A better approach is to use multiple rf sources, coming from different directions, to illuminate a larger region as homogeneously as possible, so that the reconstructed images truly reflect the differential rf absorption. The second challenge is to choose a proper ultrasound frequency for the detection. Due to the large size of a breast, typically around $15 \mathrm{~cm}$ in diameter and the increase in ultrasound attenuation at high frequencies, optimal ultrasound frequencies should be between 1 and $3 \mathrm{MHz}$, which can be initiated by rf pulses with a duration of submicrosec- onds. In addition, the selection of a proper $\mathrm{rf}$ wavelength with which to initiate the process is another key to achieving good contrast and signal-to-noise ratio.

\section{Laser-based imaging}

A tumor typically has more optical absorption than the surrounding tissues due to its high vascularity. ${ }^{28,29}$ Many experiments $^{23,70,107-109}$ have demonstrated that photoacoustic imaging is able to image blood vessels with high spatial resolution and high contrast since blood has a strong rate of light absorption compared to the surrounding tissues. For example, Oraevsky and Karabutov ${ }^{23}$ tested small-diameter vessels inside gel phantoms using a near-IR laser (1064 nm); the images demonstrated a high contrast difference, about $500 \%$, between the blood vessels and the background.

In breast imaging, near-IR light is favored because of the tissue's low absorption properties in this spectral band, which allows light to penetrate several centimeters through the tissue to reach deep tumors inside. Nonetheless, there is still enough contrast for breast cancer detection, even at the $1064 \mathrm{~nm}$ wavelength. Oraevsky et $a .^{23}$ and Esenaliev et $a l .{ }^{110}$ tested the sensitivity of PAT for detecting small model tumors embedded in bulk phantoms that simulated breast tissue. Their results demonstrated the capability of optoacoustic tomography to detect small volumes with increased absorption (dimensions: $0.6-2.0 \mathrm{~mm}$ ) at a depth of several centimeters in tissue with lower absorption using a safe level of incident laser fluence of pulsed radiation (less than $40 \mathrm{~mJ} / \mathrm{cm}^{2}$ ). $\mathrm{Ku}$ and Wang ${ }^{111}$ demonstrated that fine tubes that contain blood and ICG embedded at depths greater than $5 \mathrm{~cm}$ in chicken breast tissue could be clearly imaged by PAT with better than $780 \mathrm{~mm}$ resolution.

Oraevsky and co-workers ${ }^{23,112}$ applied what they called a laser optoacoustic imaging system (LOIS) with an arc array to the detection of breast cancer in vivo [Fig. 12(a)]. An array of optical fibers was used to deliver (to one side of the breast) light pulses of a $10 \mathrm{~ns}$ duration from a Nd:YAG (yttrium aluminum garnet) laser operated at $1064 \mathrm{~nm}$ with a $20 \mathrm{~Hz}$ repetition rate. A transducer array located on an arc surface with a $120 \mathrm{~mm}$ diameter was positioned on the opposite side of the breast to detect the PA signals. This transducer array was specially designed using piezoelectric polymer polyvinylidene fluoride (PVDF) of $110 \mu \mathrm{m}$ thickness. It had 32 elements of $1.5 \times 12 \mathrm{~mm}^{2}$ in dimension with a $4 \mathrm{~mm}$ distance between adjacent elements. Figure 12(b) shows an optoacoustic image of a breast ductal-lobular carcinoma measuring $6 \times 7 \mathrm{~mm}^{2}$ with its core $11 \mathrm{~mm}$ beneath the breast surface. The long curved features that extend downward from the tumor are artifacts that result from the limited view of detection due to the small number of detectors in this imaging system. It must be pointed out that with this transducer design, the spatial resolution in the direction perpendicular to the imaging plane, determined by the length of piezodetectors and the depth of the object, was relatively low. To improve the resolution in that direction, Kozhushko et al. ${ }^{113}$ tested another design of a linear array that consists of 32 focused elements of arc-shaped piezodetectors.

Recently, Manohar et al. ${ }^{114}$ presented what they called a photoacoustic mammoscope (PAM) for breast imaging [Fig. 


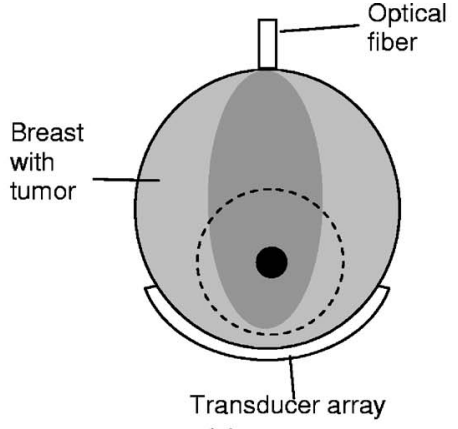

(a)

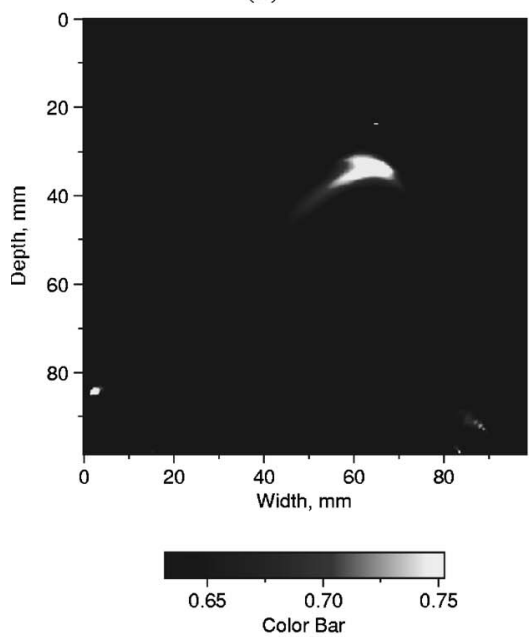

(b)

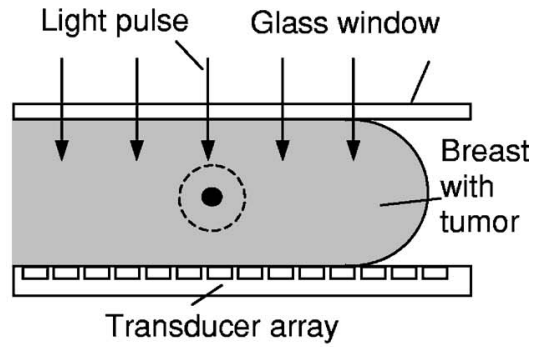

(c)

FIG. 12. (a) Side view schematic of optoacoustic imaging of a breast using an arc array. (b) Noninvasive optoacoustic image of a human breast containing a tumor. (c) Diagram of the photoacoustic mammoscope.

12(c)]. The breast was mildly compressed between a glass window and a flat detector array. The light source $(1064 \mathrm{~nm})$ was a $Q$-switched Nd:YAG laser (Brilliant-B, Quantel, Paris) with a pulse duration of $5 \mathrm{~ns}$ and a repetition rate of $10 \mathrm{~Hz}$. PA generated ultrasound propagated through the breast to be recorded by the ultrasound detector matrix at the opposite side. Their 3D image reconstruction was based on a delayand-sum beam-forming algorithm. However, the resolution attainable using PAM was not as good as that cited for the TCT scanner which is between 1 and $2 \mathrm{~mm}$ (Patent 6633774), ${ }^{101}$ nor that cited for LOIS which is $1 \mathrm{~mm} .{ }^{23}$

In summary, the most serious challenge to using PAT for breast imaging comes in selecting the proper light wavelength for the production of ultrasound. A long wavelength allows light to penetrate deeply with less attenuation, but it is not absorbed to as great a degree as light with a shorter wavelength. Fortunately, as demonstrated in many experiments, the optical contrast that exists in the near-infrared spectral range $(690-1100 \mathrm{~nm})$ between cancerous and normal tissues is still sufficient for the generation of good SNR PA images of tumors while normal tissues are depicted as a dark background. This is because the hemoglobin in blood absorbs near-infrared light very strongly and the tumor may have a concentration of blood that ranges from two to six times the amount expected in a normal breast tissue. The reason for this high blood content is the development of high blood vascularity by the tumor for the purpose of supplying its aggressive growth. Moreover, multiple optical wavelengths can be used in PAT to determine the oxygen saturation and concentration of hemoglobin, which may be used to indicate tumor malignancy, because malignant tumors are likely to be hypoxic since they consume more oxygen than normal tissues or benign tumors. In addition, a malignant lesion often has an irregular shape while a benign one is more likely to be round or elliptical. Therefore, using PAT to accurately visualize the structural detail of a mass can provide further clues in regard to malignancy.

\section{B. Small animal imaging}

Small animal models are widely used in the biological sciences, but many studies are limited by their inability to gather anatomical and physiological information noninvasively in a longitudinal manner. Therefore, functional, molecular, and morphologic quantitative imaging techniques have become important tools for providing data about biochemical, genetic, and pharmacological processes in the same animal over time in vivo.

PAT is a very attractive noninvasive imaging modality for small animal studies because it does not rely on ionizing radiation and, therefore, exhibits a clear advantage over other imaging methodologies such as $\mathrm{x}$-ray computed tomography (CT), positron emission tomography (PET), and single photon emission computed tomography (SPECT). PAT also has the critical advantage of high contrast based on light, or rf, absorption that is sensitive to tissue biochemical information, compared with other nonionizing imaging methods such as MRI, which actually images proton spins, and ultrasound imaging that relies on mechanical properties. As mentioned in the preceding text, neither pure optical imaging in deep tissues nor pure rf imaging provides good imaging resolution. On the other hand, PAT can achieve better than submillimeter resolution in centimeters of depth.

Additionally, PAT images can be acquired relatively quickly. Compared with traditional histological techniques, which are quite time consuming (taking several days to weeks), PAT images can be acquired in three-dimensional data sets with a very accurate depiction of a sample in a relatively short amount of time (minutes to a few hours) or potentially at a video rate with advanced hardware for data acquisition and image reconstruction or display. Most importantly, images can be acquired in vivo, allowing for the longitudinal acquisition of anatomical and physiological information from the same subject.

We have discussed some applications of PAT in the preceding sections, such as PA microcopy, which can be easily adopted to image superficial tumors in small animal models. 


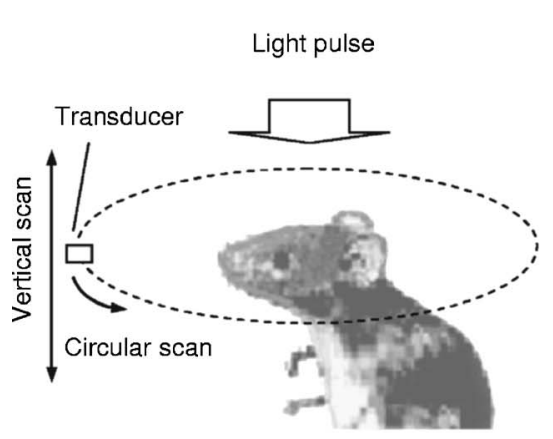

(a)

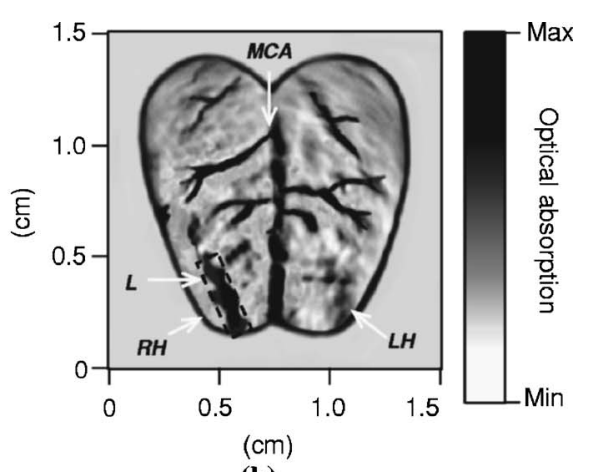

(b)
FIG. 13. (a) Diagram of photoacoustic imaging system for small animals in a circular or cylindrical scan. (b) A cross-sectional photoacoustic image of a rat brain. $\mathrm{RH}$, right cerebral hemisphere; LH, left cerebral hemisphere; $\mathrm{L}$, lesion; MCA, middle cerebral artery (Ref. 116).
In Secs. VII B 1, VII B 2, and VII B 3 additional examples are provided to illustrate other potential applications of PAT in small animal studies.

\section{Structure imaging}

a. Brain and tumor imaging. Wang et al. ${ }^{115,116}$ demonstrated PAT imaging of small animal brains in a circular scan measurement configuration [Fig. 13(a)]. A $Q$-switched $\mathrm{Nd}$ :YAG laser was adopted to provide $532 \mathrm{~nm}$ laser pulses with a $6.5 \mathrm{~ns}$ pulse duration and a $10 \mathrm{~Hz}$ repetition frequency. The laser beam was expanded and homogenized to provide an incident energy density of less than $10 \mathrm{~mJ} / \mathrm{cm}^{2}$ on the skin of the rat head. The ultrasound was coupled into a high-sensitivity ultrasound transducer (V383, Panametrics, $3.5 \mathrm{MHz}$ ) through water in the assay tank. Using a $532 \mathrm{~nm}$ wavelength, blood vessels in the cortical surface of small animals were imaged transcranially with the skin and the skull intact [Fig. 13(b)]. ${ }^{115}$ The contrast was quite high at this wavelength; however, the imaging depth was limited to $1 \mathrm{~cm}$ or so in length. Nevertheless, this depth was enough for imaging the entire brain of a small animal such as a mouse. Not only blood vessels but also other detailed brain structures, such as the cerebellum, hippocampus, and ventriculi lateralis, were clearly imaged by a small aperture transducer (XMS-310, Panametrics, $10.4 \mathrm{MHz}$, active element $2 \mathrm{~mm}$ ) in a scan radius of $2.8 \mathrm{~cm} .{ }^{116}$

Later, using a similar system, $\mathrm{Ku}$ et $a .^{21,117}$ reported studies of rat brain tumors using multiple-bandwidth photoacoustic tomography, in which the angiogenesis associated with the tumors was clearly revealed both in vitro and in vivo. In summary, PAT is suitable for monitoring tumor growth, angiogenesis, and antiangiogenic therapy in experimental carcinogenesis on animal models. Further, this noninvasive technology allows for monitoring the same animal at multiple times as well as reducing the individual variability and the number of experimental animals required.

b. Body imaging. Kruger et al. designed small-animal PAT imaging systems with an ultrasound linear array, ${ }^{118}$ or arc array, ${ }^{119}$ utilizing near IR light to enhance the imaging depth at the expense of reduced contrast. (See the diagram of the system with an arc array in Fig. $14 .{ }^{119}$ ) The array, consisting of 128 elements, was laid out on a cylindrical surface with a $40 \mathrm{~mm}$ radius of curvature. Each element had a size of $1.8 \times 2.0 \mathrm{~mm}^{2}$ and a peak response at $2.5 \mathrm{MHz}$. The distribution of the elements was chosen to provide an approximately uniform coverage over the surface of a sphere when it was rotated to a plurality of angles about the vertical axis. The sample was placed inside a thin-walled plastic tube, which was affixed to a computer-controlled rotary stage (not shown), and was immersed in the scan tank for imaging. Pulsed light ( $<10 \mathrm{~ns}, \sim 25 \mathrm{~mJ} /$ pulse $)$ from a Nd:YAG or a tunable optical parametric oscillator (OPO) laser was delivered to the scan tank through a set of four fiber optic bundles (not shown). The vertical positioning of the sample in the tube was controlled by a linear stage (not shown). Nude mice were imaged ex vivo. ${ }^{119}$ From the images, vascular anatomy could be isolated from nonvascular anatomy without the use of cardiovascular contrast agents as used routinely in X-ray CT and MRI. Drastic differences in the appearance of the mouse anatomy were observed when imaged at $1064 \mathrm{~nm}$ compared to $800 \mathrm{~nm}$. At $1064 \mathrm{~nm}$, optical absorption was dominated by the presence of water; at $800 \mathrm{~nm}$, water absorption was virtually nil, and hemoglobin in the blood dominated optical absorption. The study indicates that multiple-wavelength PAT images can reveal different details related to the optical absorption preferences of the tissues.

\section{Functional imaging}

PAT imaging techniques based on optical contrast analysis have the potential to visualize dynamic and functional properties of the nervous system via optical signals resulting from changes in blood volume, oxygen consumption, and cellular swelling associated with brain physiology and pathology.

Wang et al. ${ }^{116}$ demonstrated a PAT system [Fig. 13(a)] for visualizing the functional representations of whisker stimulations in the cerebral cortex of rats (Fig. 15). PAT images of the rat superficial cortex were taken with, and without, stimulating the left or right whiskers, respectively. Subtracting the PAT image without whisker stimulation from the

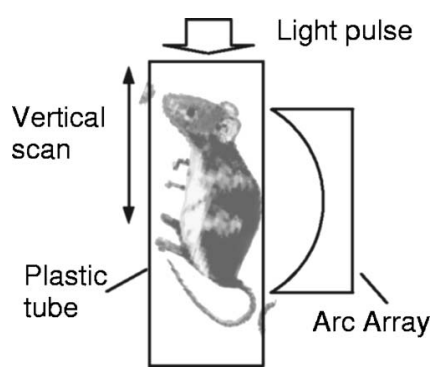

FIG. 14. Diagram of photoacoustic imaging system for small animals using arc array. 


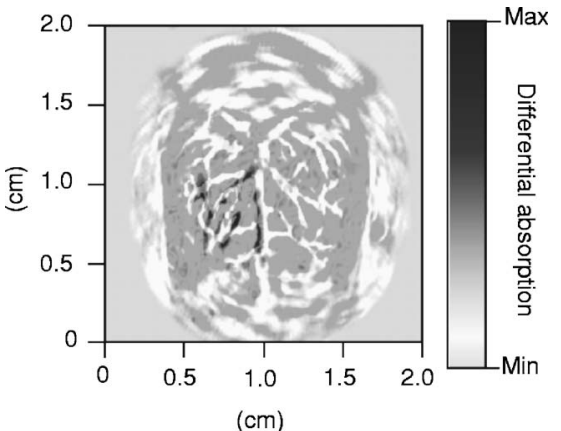

FIG. 15. Noninvasive functional photoacoustic image corresponding to the left-side whisker stimulations, acquired with the skin and skull intact (Ref. 116).

two PAT images with whisker stimulation produced two maps of functions evoked by these stimuli. These images clearly revealed functional cerebral homodynamic changes in response to whisker stimulation on either side of the rat snout. In addition, Wang et al. also successfully imaged hyperoxia- and hypoxia-induced cerebral hemodynamic changes ${ }^{116}$ and an angiography of animal brains in vivo with near-IR light and an optical contrast agent. ${ }^{120}$

\section{Molecular imaging}

PAT can be readily extended to the molecular imaging of pathologically changed tissues, such as cancer cells, with the implementation of optical absorbent biomarkers or targeted molecular probes to enhance the absorption contrast of diseased tissue areas, if the absorption contrast agents are properly conjugated to bioactive peptides, proteins, antibodies, hormones, drugs, or other bioactive agents.

Kruger et al. ${ }^{119}$ proposed a dual-wavelength strategy to isolate dye-labeled molecular probes. Their phantom experiments demonstrated that by using dual-wavelength subtraction, PAT was able to detect a concentration of 5 fmol or less of a near-IR dye, ICG, in a $1 \mu \mathrm{l}$ volume. Wang et al. ${ }^{120}$ tested another near-IR dye, indocyanine green polyethylene glycol (ICG-PEG), in living rats and found that this dye had an obviously slower clearance than the native ICG. In addition, the feasibility of using gold nanoparticles ${ }^{121,122}$ or nanoshells $^{123}$ in vivo as new contrast-enhancing agents for photoacoustic tomography was also reported.

Compared to pure optical molecular imaging methods, such as fluorescence imaging and diffusion optical tomography, PAT has significantly higher spatial resolution.

\section{Other experiments}

Many other interesting experiments have been reported recently. For example, Larin et al. ${ }^{124}$ studied optoacoustic laser monitoring of the cooling and freezing of tissues in cryotherapy and in cryobiology. Their study showed that the amplitude of PA signals increased sharply upon freezing; hence, the PA technique has the potential for real-time monitoring of temperature variation and frozen-zone formation. Pilatou et al. ${ }^{125}$ presented an analysis of 3D photoacoustic imaging of a vascular tree from a Wistar rat in vitro. Esenaliev et al. ${ }^{126}$ reported a feasibility study for an optoacoustic technique for the noninvasive monitoring of blood oxy- genation, and Petrov et al. ${ }^{127}$ conducted an in vivo study monitoring cerebral blood oxygenation in sheep. Siphanto et $a l{ }^{128}$ conducted a study on serial noninvasive photoacoustic imaging of neovascularization in tumor angiogenesis, and the results demonstrated the potential of the method as a tool for tumor angiogenesis research in small animals. More experiments can be found in recent proceedings. ${ }^{13-17}$

\section{SUMMARY AND DISCUSSION}

PAT combines good acoustic resolution with optical or $\mathrm{rf}$ absorption contrast. It is suitable for biological tissues with inhomogeneous optical or rf absorption but relatively homogeneous acoustical properties, while ultrasound imaging depends primarily on acoustic heterogeneity. Since PA signals are excited internally by EM absorption and are propagated one way to the detectors, small speed variations do not much affect the travel time of the sound in a finite-length path. Hence, PAT has better tolerance to sound speed variation than conventional pulse-echo ultrasound imaging which detects round-trip ultrasound.

The imaging depth and spatial resolution of PAT in soft tissues are scalable with the acoustic frequency. rf-based PAT using submicrosecond pulse excitation has been shown to have submillimeter resolution even multiple centimeters into soft tissue due to the weak attenuation of low-megahertz ultrasound and the good penetration of $\mathrm{rf}$ waves. Laser-based PAT using nanosecond-pulse excitation can obtain high resolution up to $50 \mu \mathrm{m}$ or better only a few millimeters into soft tissue because of the strong attenuation of high-frequency ultrasound; nevertheless, it can also image up to several centimeters into soft tissue at the cost of resolution. PA imaging is also free of speckle effect, whereas conventional pulseecho ultrasound imaging has strong speckle artifacts.

At the same time, PAT inherits several limitations from both acoustic and EM radiations. Hard tissues, such as the human skull, produce strong ultrasonic wave-front aberrations that would hinder the application of PAT to, for example, human brain imaging. Contact measurements are often required for acoustic coupling in PAT. Although light can penetrate into soft tissues up to centimeters deep, the light attenuation accentuated by strong scattering is significant and the dynamic range of the PA signal may be extensive, which makes it difficult to image deeply buried small tumors. Two possible corrections can potentially improve PAT's image quality. One is, after combining pure rf or optical imaging with PAT, to use the fluence distribution in tissues extracted from the rf or optical image to normalize the rf or optical absorption reconstructed from the PAT. The other is to combine pure ultrasound imaging with PAT, and then implement any ultrasound inhomogeneity, such as speed variation, into the PAT reconstruction algorithm. We expect to see reliable algorithms and experimental demonstrations of these ideas in the near future.

PAT can be used to image animal or human organs, such as the breast and the brain, where the angiogenesis networks, blood vessels, and blood perfusion can be measured. With the implementation of an ultrasound detection array, PAT can potentially be applied in vivo for real-time imaging. The 
same array can also produce conventional ultrasound images for the comparison and codisplay of two types of contrast. In addition, a sample can be imaged with the excitation of different EM wavelengths of interest. Multiple optical wavelengths can be used to extract physiological parameters such as hemoglobin concentrations and oxygenation states. Molecular contrast agents will make it feasible to use PAT for molecular imaging, such as imaging gene expression and endogenous molecular processes, in which the achievable spatial resolution in the study of deep structures is better than in the all-optically-based approaches. PAT is a potential tool for monitoring various biological parameters since PA signals are sensitive to the EM, thermal, and elastic properties of biological tissue samples.

In conclusion, PA imaging is still in its infancy and there have as yet been no large clinical trials, although many initial studies have demonstrated the possibilities for applications in the biomedical field. Clearly, we should expect to see many exciting clinical applications of PA technologies in the near future.

\section{ACKNOWLEDGMENTS}

This project was sponsored in part by the U.S. Army Medical Research and Materiel Command Grant No. DAMD17-00-1-0455, the National Institute of Health Grant Nos. R01 EB000712 and R01 NS46214, and Texas Higher Education Coordinating Board Grant No. ARP 000512-00632001.

${ }^{1}$ A. G. Bell, Am. J. Sci. 20, 305 (1880).

${ }^{2}$ C. K. N. Patel and A. C. Tam, Rev. Mod. Phys. 53, 517 (1981).

${ }^{3}$ G. A. West, J. J. Barrett, D. R. Siebert, and K. V. Reddym, Rev. Sci. Instrum. 54, 797 (1983).

${ }^{4}$ D. A. Hutchins and A. C. Tam, IEEE Trans. Ultrason. Ferroelectr. Freq. Control 33, 429 (1986).

${ }^{5}$ A. C. Tam, Rev. Mod. Phys. 58, 381 (1986).

${ }^{6}$ M. W. Sigrist, J. Appl. Phys. 60, R83 (1986).

${ }^{7}$ A. Rosencwaig, Photoacoustics and Photoacoustic Spectroscopy (Wiley, New York, 1980).

${ }^{8}$ V. P. Zharov and V. S. Letokhov, Laser Optoacoustic Spectroscopy, Springer Series in Optical Science Vol. 37 (Springer-Verlag, New York, 1986).

${ }^{9}$ Principles and Perspectives of Photothermal and Photoacoustic Phenomena, edited by A. Mandelis (Elsevier Science, New York, 1992).

${ }^{10}$ K. H. Michaelian, Photoacoustic Infrared Spectroscopy (WileyInterscience, Hoboken, NJ, 2003).

${ }^{11}$ J. F. McClelland, R. W. Jones, and S. J. Bajic, in Handbook of Vibrational Spectroscopy, edited by J. M. Chalmers and P. R. Griffiths (Wiley, New York, 2002).

${ }^{12}$ V. E. Gusev and A. A. Karabutov, Laser Optoacoustics (AIP, New York, 1993).

${ }^{13}$ Proc. SPIE 3916 (2000).

${ }^{14}$ Proc. SPIE 4256 (2001).

${ }^{15}$ Proc. SPIE 4618 (2002).

${ }^{16}$ Proc. SPIE 4960 (2003).

${ }^{17}$ Proc. SPIE 5320 (2004); 5697 (2005).

${ }^{18}$ F. A. Duck, Physical Properties of Tissue (Academic, London, 1990).

${ }^{19}$ L. E. Larsen and J. H. Jacobi, Medical Applications of Microwave Imaging (IEEE, New York, 1986).

${ }^{20}$ P. M. Meaney, M. W. Fanning, D. Li, S. P. Poplack, and K. D. Paulsen, IEEE Trans. Microwave Theory Tech. 48, 1841 (2000).

${ }^{21}$ G. Ku, X. Wang, G. Stoica, and L.-H. Wang, Phys. Med. Biol. 49, 1329 (2004).

${ }^{22}$ A. A. Oraevsky and A. A. Karabutov, in Handbook of Optical Biomedical Diagonstics, edited by V. V. Tuchin (SPIE, Bellingham, WA, 2002), Chap. 10.
${ }^{23}$ A. A. Oraevsky and A. A. Karabutov, in Biomedical Photonics Handbook, edited by T. Vo-Dinh (CRC, Boca Raton, FL, 2003), Chap. 34.

${ }^{24}$ W. F. Cheong, S. A. Prahl, and A. J. Welch, IEEE J. Quantum Electron. 26, 2166 (1990).

${ }^{25}$ J. Mobley and T. Vo-Dinh, in Biomedical Photonics Handbook, edited by T. Vo-Dinh (CRC, Boca Raton, FL, 2003), Chap. 2.

${ }^{26}$ K. R. Foster, IEEE Trans. Plasma Sci. 28, 15 (2000).

${ }^{27}$ C. C. Johnson and A. W. Guy, Proc. IEEE 60, 692 (1972).

${ }^{28}$ N. Weidner, J. P. Semple, W. R. Welch, and J. Folkman, N. Engl. J. Med. 324, 1 (1991).

${ }^{29}$ J. Folkman, Nat. Med. 1, 27 (1995).

${ }^{30}$ The Institute of Electrical and Electronics Engineers, IEEE Standard for Safety Levels with Respect to Human Exposure to Radio Frequency Electromagnetic Fields, $3 \mathrm{kHz}$ to $300 \mathrm{GHz}$ : IEEE Std C95.1 (IEEE, Inc., New York, 1999).

${ }^{31}$ American National Standards Institute, American National Standard for the Safe Use of Lasers in Health Care Facilities: Standard Z136.1-2000 (ANSI, Inc., New York, 2000).

${ }^{32}$ A. L. McKenzie, Phys. Med. Biol. 35, 1175 (1990).

${ }^{33}$ P. N. T. Wells, Rep. Prog. Phys. 62, 671 (1999).

${ }^{34}$ V. G. Andreev, A. A. Karabutov, and A. A. Oraevsky, IEEE Trans. Ultrason. Ferroelectr. Freq. Control 50, 1383 (2003).

${ }^{35}$ P. C. Beard, F. Pérennès, E. Draguioti, and T. N. Mills, Opt. Lett. 23, 1235 (1998).

${ }^{36}$ P. C. Beard, F. Pérennès, and T. N. Mills, IEEE Trans. Ultrason. Ferroelectr. Freq. Control 46, 1575 (1999).

${ }^{37}$ G. Paltauf, H. Schmidt-Kloiber, K. P. Köstli, and M. Frenz, Appl. Phys. Lett. 75, 1048 (1999).

${ }^{38}$ J. J. Niederhauser, D. Frauchiger, H. P. Weber, and M. Frenz, Appl. Phys. Lett. 81, 571 (2002).

${ }^{39}$ S. Ashkenazi, Y. G. Hou, T. Buma, and M. O'Donnell, Appl. Phys. Lett. 86, 134102 (2005).

${ }^{40}$ S. A. Carp, A. Guerra, III, S. Q. Duque Jr., and V. Venugopalana, Appl. Phys. Lett. 85, 5772 (2004).

${ }^{41}$ P. C. Beard, IEEE Trans. Ultrason. Ferroelectr. Freq. Control 52, 1002 (2005).

${ }^{42}$ A. A. Karabutov, N. B. Podymova, and V. S. Letokhov, Appl. Phys. B: Lasers Opt. 63, 545 (1996).

${ }^{43}$ A. A. Oraevsky, S. L. Jacques, and F. K. Tittel, Appl. Opt. 36, 402 (1997).

${ }^{44}$ A. A. Karabutov, E. V. Savateeva, N. B. Podymova, and A. A. Oraevsky, J. Appl. Phys. 87, 2003 (2000).

${ }^{45}$ G. Paltauf and H. Schmidt-Kloiber, J. Appl. Phys. 88, 1624 (2000).

${ }^{46}$ K. P. Köstli, M. Frenz, H. P. Weber, G. Paltauf, and H. Schmidt-Kloiber, J. Appl. Phys. 88, 1632 (2000).

${ }^{47}$ J. A. Viator, S. L. Jacques, and S. A. Prahl, IEEE J. Sel. Top. Quantum Electron. 5, 989 (1999).

${ }^{48}$ J. A. Viator, G. Au, G. Paltauf, S. L. Jacques, S. A. Prahl, H. Ren, Z. Chen, and J. S. Nelson, Lasers Surg. Med. 30, 141 (2002).

${ }^{49}$ See "Ultrasonic transducer technical note," in http://www.panametricsndt.com

${ }^{50}$ G. Ku and L.-H. Wang, Med. Phys. 27, 1195 (2000).

${ }^{51}$ G. Ku and L.-H. Wang, Med. Phys. 28, 4 (2001).

${ }^{52}$ M.-H. Xu, G. Ku, and L.-H. Wang, Med. Phys. 28, 1958 (2001).

${ }^{53}$ R. A. Kruger and P. Liu, Med. Phys. 21, 1179 (1994).

${ }^{54}$ A. A. Karabutov, E. V. Savateeva, and A. A. Oraevsky, Laser Phys. 13, 711 (2003).

${ }^{55}$ K. Maslov, G. Stoica, and L.-H. Wang, Opt. Lett. 30, 625 (2005).

${ }^{56}$ C. Guittet, F. Ossant, L. Vaillant, and M. Berson, IEEE Trans. Biomed. Eng. 46, 740 (1999).

${ }^{57}$ J. J. Niederhauser, M. Jaeger, and M. Frenz, Appl. Phys. Lett. 85, 846 (2004).

${ }^{58}$ M. Haltmeier, O. Scherzer, P. Burgholzer, and G. Paltauf, Inverse Probl. 20, 1663 (2004).

${ }^{59}$ G. J. Diebold, T. Sun, and M. I. Khan, Phys. Rev. Lett. 67, 3384 (1991).

${ }^{60}$ J. D. Jackson, Classical Electrodynamics, 2nd ed. (Wiley, New York, 1999).

${ }^{61}$ G. B. Arfken and H. J. Weber, Mathematical Methods for Physicists, 4th ed. (Academic, San Diego, CA, 1995).

${ }^{62} \mathrm{P}$. M. Morse and H. Feshbach, Methods of Theoretical Physics (McGrawHill, New York, 1953).

${ }^{63}$ R. A. Kruger, P. Liu, Y. R. Fang, and C. R. Appledorn, Med. Phys. 22, 1605 (1995).

${ }^{64}$ R. Y. Fang, R. A. Kruger, C. R. Appledorn, and P. Liu, Prog. Nat. Sci. S6, S-598 (1996). 
${ }^{65}$ R. A. Kruger, D. R. Reinecke, and G. A. Kruger, Med. Phys. 26, 1832 (1999).

${ }^{66}$ P. Liu, Phys. Med. Biol. 43, 667 (1998).

${ }^{67}$ V. G. Andreev, D. A. Popov, D. V. Sushko, A. A. Karabutov, and A. A. Oraevsky, Proc. SPIE 4256, 119 (2001).

${ }^{68}$ V. G. Andreev, D. A. Popov, D. V. Sushko, A. A. Karabutov, and A. A. Oraevsky, Proc. SPIE 4618, 137 (2002).

${ }^{69}$ Y. Xu, L.-H. Wang, G. Ambartsoumian, and P. Kuchment, Med. Phys. 31, 724 (2004).

${ }^{70}$ C. G. A. Hoelen, F. F. M. de Mul, R. Pongers, and A. Dekker, Opt. Lett. 23, 648 (1998).

${ }^{71}$ C. G. A. Hoelen and F. F. M. de Mul, Appl. Opt. 39, 5872 (2000).

${ }^{72}$ K. P. Köstli, M. Frenz, H. P. Weber, G. Paltauf, and H. Schmidt-Kloiber, Appl. Opt. 40, 3800 (2001).

${ }^{73}$ D. Feng, Y. Xu, G. Ku, and L.-H. Wang, Med. Phys. 28, 2427 (2001).

${ }^{74}$ C. K. Liao, M. L. Li, and P. C. Li, Opt. Lett. 29, 2506 (2004).

${ }^{75}$ B.-Z. Yin, D. Xing, Y. Wang, Y.-G. Zeng, Y. Tan, and Q. Chen, Phys. Med. Biol. 49, 1339 (2004).

${ }^{76}$ M. Xu and L.-H. Wang, IEEE Trans. Med. Imaging 21, 814 (2002).

${ }^{77}$ M. Xu, Y. Xu, and L.-H. Wang, IEEE Trans. Biomed. Eng. 50, 1086 (2003).

${ }^{78}$ G. Paltauf, J. A. Viator, S. A. Prahl, and S. L. Jacques, J. Acoust. Soc. Am. 112, 1536 (2002).

${ }^{79}$ Y. Xu and L.-H. Wang, IEEE Trans. Ultrason. Ferroelectr. Freq. Control 50, 1134 (2003).

${ }^{80}$ Y. V. Zhulina, Appl. Opt. 39, 5971 (2000).

${ }^{81}$ M. A. Anastasio, J. Zhang, X.-C. Pan, Y. Zou, G. Ku, and L.-H. Wang, IEEE Trans. Med. Imaging 24, 199 (2005).

${ }^{82}$ J. Zhang, M. A. Anastasio, X.-C. Pan, and L.-H. Wang, IEEE Trans. Med. Imaging 24, 817 (2005)

${ }^{83}$ S. J. Norton and M. Linzer, IEEE Trans. Biomed. Eng. 28, 202 (1981).

${ }^{84}$ S. J. Norton, J. Acoust. Soc. Am. 67, 1266 (1980).

${ }^{85}$ Y. Xu, D. Feng, and L.-H. Wang, IEEE Trans. Med. Imaging 21, 823 (2002).

${ }^{86}$ K. P. Köstli, D. Frauchiger, J. J. Niederhauser, G. Paltauf, H. P. Weber, and M. Frenz, IEEE J. Sel. Top. Quantum Electron. 7, 918 (2001).

${ }^{87}$ K. P. Köstli, M. Frenz, H. Bebie, and H. P. Weber, Phys. Med. Biol. 46, 1863 (2001).

${ }^{88}$ A. C. Kak and M. Slaney, Principles of Computerized Tomographic Imaging (IEEE, New York, 1999).

${ }^{89}$ S. J. Norton, J. Acoust. Soc. Am. 67, 853 (1980).

${ }^{90}$ Y. Xu, M.-H. Xu, and L.-H. Wang, IEEE Trans. Med. Imaging 21, 829 (2002).

${ }^{91}$ S. J. Norton and T. Vo-Dinh, J. Opt. Soc. Am. 20, 1859 (2003).

${ }^{92}$ D. V. Finch, S. K. Patch, and Rakesh, SIAM J. Math. Anal. 35, 1213 (2003).

${ }^{93}$ M.-H. Xu and L.-H. V. Wang, Phys. Rev. E 71, 016706 (2005).

${ }^{94}$ Y. Xu and L.-H. Wang, Phys. Rev. Lett. 92, 033902 (2004).

${ }^{95}$ X. Pan, Y. Zou, and M. Anastasio, IEEE Trans. Image Process. 12, 784 (2003).

${ }^{96}$ S. K. Patch, Phys. Med. Biol. 49, 2305 (2004).

${ }^{97}$ M.-H. Xu and L.-H. V. Wang, Phys. Rev. E 67, 056605 (2003).

${ }^{98}$ M.-H. Xu and L.-H. V. Wang, Med. Phys. 29, 1661 (2002).

${ }^{99}$ By the Committee on Technologies for the Early Detection of Breast Cancer, Mammography and Beyond: Developing Technologies for the Early Detection of Breast Cancer, edited by S. J. Nass, I. C. Henderson, and J. C. Lashof (National Academies, Washington, 2001).

${ }^{100}$ S. Chaudhary, R. Mishra, A. Swarup, and J. Thomas, Indian J. Biochem.
Biophys. 21, 76 (1984).

${ }^{101}$ OptoSonics, Patent Nos. 5713356, 6102857, 6104942, 6216025B1, 6292682, 6490470, and 6633774 (pending).

${ }^{102}$ R. A. Kruger, K. K. Kopecky, A. M. Aisen, D. R. Reinecke, G. A. Kruger, and W. L. Kiser, Jr., Radiology 211, 275 (1999).

${ }^{103}$ R. A. Kruger, K. D. Miller, H. E. Reynolds, W. L. Kiser, Jr., D. R. Reinecke, and G. A. Kruger, Radiology 216, 279 (2000).

${ }^{104}$ R. A. Kruger, K. M. Stantz, and W. L. Kiser, Jr., Proc. SPIE 4682, 521 (2002).

${ }^{105}$ M.-H. Xu, G. Ku, X. Jin, L.-H. Wang, B. D. Fornage, and K. K. Hunt, Proc. SPIE 5697, 45 (2005).

${ }^{106}$ G. Ku, B. D. Fornage, X. Jin, M.-H. Xu, K. K. Hung, and L.-H. V. Wang, Technol. Cancer Res. Treat. 4, 559 (2005).

${ }^{107}$ C. G. A. Hoelen and F. F. M. de Mul, Appl. Opt. 39, 5872 (2000).

${ }^{108}$ X. Wang, Y. Xu, M. Xu, S. Yokoo, E. S. Fry, and L.-H. Wang, Med. Phys. 29, 2799 (2002).

${ }^{109}$ R. G. M. Kolkman, E. Hondebrink, W. Steenbergen, and F. F. M. de Mul, IEEE J. Sel. Top. Quantum Electron. 9, 343 (2003).

${ }^{110}$ R. O. Esenaliev, A. A. Karabutov, and A. A. Oraevsky, IEEE J. Sel. Top. Quantum Electron. 5, 981 (1999).

${ }^{111}$ G. Ku and L.-H. Wang, Opt. Lett. 30, 507 (2005).

${ }^{112}$ A. A. Oraevsky, A. A. Karabutov, S. V. Solomatin, E. V. Savateeva, V. G. Andreev, Z. Gatalica, H. Singh, and R. D. Fleming, Proc. SPIE 4256, 6 (2001).

${ }^{113}$ V. Kozhushko, T. Khokhlova, A. Zharinov, I. Pelivanov, V. Solomatin, and A. Karabutov, J. Acoust. Soc. Am. 116, 1498 (2004).

${ }^{114}$ S. Manohar, A. Kharine, J. C. G. van Hespen, W. Steenbergen, and T. G. van Leeuwen, Phys. Med. Biol. 50, 2543 (2005).

${ }^{115}$ X. Wang, Y. Pang, G. Ku, G. Stoica, and L.-H. V. Wang, Opt. Lett. 28, 1739 (2003).

${ }^{116}$ X. Wang, Y.-J. Pang, G. Ku, X.-Y. Xie, G. Stoica, and L.-H. V. Wang, Nat. Biotechnol. 21, 803 (2003).

${ }^{117}$ G. Ku, X. Wang, X. Xie, G. Stoica, and L.-H. Wang, Appl. Opt. 44, 770 (2005).

${ }^{118}$ R. A. Kruger, W. L. Kiser, Jr., D. R. Reinecke, and G. A. Kruger, Med. Phys. 30, 856 (2003).

${ }^{119}$ R. A. Kruger, W. L. Kiser, Jr., D. R. Reinecke, G. A. Kruger, and K. D. Miller, Molecular Imaging 2, 113 (2003).

${ }^{120}$ X. Wang, G. Ku, M. A. Wegiel, D. J. Bornhop, G. Stoica, and L.-H. Wang, Opt. Lett. 29, 730 (2004).

${ }^{121}$ M. A. Eghtedari, J. A. Copland, V. L. Popove, N. A. Kotov, M. Motamedi, and A. A. Oraevsky, Proc. SPIE 4960, 76 (2003).

${ }^{122}$ J. A. Copland, M. Eghtedari, V. L. Popov, N. Kotov, N. Mamedova, M. Motamedi, and A. A. Oraevsky, Mol. Imag. Biol. 6, 341 (2004).

${ }^{123}$ Y. Wang, X. Xie, X. Wang, G. Ku, K. L. Gill, D. P. O'Neal, G. Stoica, and L.-H. Wang, Nano Lett. 4, 1689 (2004).

${ }^{124}$ K. V. Larin, I. V. Larina, M. Motamedi, and R. O. Esenaliev, Quantum Electron. 32, 953 (2002).

${ }^{125}$ M. C. Pilatou, R. I. Siphanto, L. N. A. van Adrichem, and F. F. M. de Mul, Rev. Sci. Instrum. 74, 384 (2003).

${ }^{126}$ R. O. Esenaliev, I. V. Larina, K. V. Larin, D. J. Deyo, M. Motamedi, and D. S. Prough, Appl. Opt. 41, 4722 (2002).

${ }^{127}$ Y. Y. Petrov, D. S. Prough, D. J. Deyo, M. Klasing, M. Motamedi, and R. O. Esenaliev, Anesthesiology 102, 69 (2005).

${ }^{128}$ R. I. Siphanto, K. K. Thumma, R. G. M. Kolkman, T. G. van Leeuwen, F. F. M. de Mul, J. W. van Neck, L. N. A. van Adrichem, and W. Steenbergen, Opt. Express 13, 89 (2005). 